\title{
Monte Carlo studies of geomagnetic field effects on the imaging air Cherenkov technique for the MAGIC telescope site
}

\author{
S.C. Commichau ${ }^{\mathrm{a}, *}$, A. Biland ${ }^{\mathrm{a}}$, J.L. Contreras ${ }^{\mathrm{b}}$, R. de los Reyes ${ }^{\mathrm{b}}$, A. Moralejo ${ }^{\mathrm{c}}$, \\ J. Sitarek ${ }^{\mathrm{d}, \mathrm{e}}$, D. Sobczyńska ${ }^{\mathrm{e}}$ \\ ${ }^{\text {a }}$ ETH Zurich, $\mathrm{CH}-8093$ Switzerland \\ ' Universidad Complutense, E-28040 Madrid, Spain \\ c Institut de Física d'Altes Energies, Edifici Cn., E-08193 Bellaterra, Barcelona, Spain \\ ${ }^{\mathrm{d}}$ Max-Planck-Institut für Physik, D-80805 München, Germany \\ e University of Łódź, PL-90236 Lodz, Poland
}

\section{On behalf of the MAGIC collaboration ${ }^{1}$}

\section{A R T I C L E I N F O}

\section{Article history:}

Received 20 May 2008

Received in revised form

16 July 2008

Accepted 17 July 2008

Available online 3 August 2008

Keywords:

Extensive air showers

Monte Carlo simulations

$\gamma$-ray

Geomagnetism

\begin{abstract}
A B S T R A C T
Imaging air Cherenkov telescopes (IACTs) detect the Cherenkov light from extensive air showers (EAS) initiated by very high energy (VHE) $\gamma$-rays impinging on the Earth's atmosphere. Due to the overwhelming background from hadron-induced EAS, the discrimination of the rare $\gamma$-like events is vital. The influence of the geomagnetic field (GF) on the development of EAS can further complicate the imaging air Cherenkov technique. The amount and the angular distribution of Cherenkov light from EAS can be obtained by means of Monte Carlo (MC) simulations. Here we present the results from dedicated MC studies of GF effects on images from $\gamma$-ray initiated EAS for the MAGIC telescope site, where the GF strength is $\sim 40 \mu \mathrm{T}$. The results from the MC studies suggest that GF effects degrade not only measurements of very low energy $\gamma$-rays below $\sim 100 \mathrm{GeV}$ but also those at TeV-energies.
\end{abstract}

(c) 2008 Elsevier B.V. All rights reserved.

\section{Introduction}

Imaging air Cherenkov telescopes (IACTs) aim at the detection of Cherenkov light from extensive air showers (EAS) initiated by very high energy (VHE) $\gamma$-rays impinging on the Earth's atmosphere. IACTs make use of the differences between the angular distributions of Cherenkov light from $\gamma$-ray and hadron-induced EAS to efficiently discriminate the hadronic background. Due to the overwhelming abundance of hadrons in the cosmic rays (mostly protons), the discrimination of the rare $\gamma$-ray events is rather difficult, in particular for energies below $100 \mathrm{GeV}$. Only a small fraction of the recorded data are due to $\gamma$-ray initiated EAS. The influence of the geomagnetic field (GF) on the development of EAS can further complicate the suppression of the hadronic background and therefore reduces the sensitivity of an instrument. In addition, GF effects can systematically affect the energy resolution of an IACT [1-3].

\footnotetext{
* Corresponding author. Tel.: +414463 32185; fax: +41446331104. E-mail address: sebastian.commichau@phys.ethz.ch (S.C. Commichau).

${ }^{1}$ http://wwwmagic.mppmu.mpg.de/collaboration/members/index.html
}

The influence of the GF on EAS was already qualitatively discussed in 1953 [4]. It was pointed out that the east-west separation of electrons and positrons in EAS due to the GF can be non-negligible compared to the displacement from multiple Coulomb scattering. Furthermore, the Lorentz force systematically deflects the particles into opposite directions whereas the displacement due to multiple Coulomb scattering is random. It was also argued that GF effects are relatively less important for hadron-induced EAS than for $\gamma$-ray-induced EAS. The scattering angles occurring in nuclear interactions of hadronic EAS give rise to a lateral displacement of the shower particles much larger than that due to the influence of the GF. The comparatively large transverse momenta of the secondary particles result in a large angular spread of the directions of the electromagnetic subcascades generated by pion decay.

The influence of the GF on the average lateral spread of atmospheric Cherenkov radiation was studied by means of computer simulations already more than 30 years ago [5]. GF effects on real $\gamma$-ray and proton initiated EAS were later on studied using a non-imaging Cherenkov telescope [6]. It was reported that the influence of the GF on proton initiated EAS results in a significant reduction of the count rate. It was shown elsewhere [7] that IACT measurements of TeV $\gamma$-rays from the Crab nebula were 
not significantly affected when the component of the GF normal to the shower axis, i.e. transversal component of the GF, was below $35 \mu \mathrm{T}$. The average shape and reconstructed intensity of Cherenkov images from hadrons was found to be independent of the transversal component of the GF. However, it was pointed out that the instrument was not sensitive enough to study GF effects. More recent measurements of $\gamma$-ray showers carried out with a transversal component of the GF strength of $\left|\vec{B}_{\perp}\right|>40 \mu \mathrm{T}$ revealed GF effects in observational data compatible to those predicted by MC simulations, both for $\gamma$-ray and hadron showers $[8,9]$. The authors suggest that for EAS developing under unfavourable orientation with respect to the direction of the GF the corresponding Cherenkov light images in the camera of an IACT will be rotated. As the information on the orientation of shower images provides the most powerful discrimination between $\gamma$-ray shower images from a point-like source and any unwanted isotropic background (mainly due to hadrons) this results in a degradation of the sensitivity of an IACT. However, it was demonstrated that a correction for GF effects in $\gamma$-ray initiated Cherenkov images is possibly resulting in an increased detection significance and better sensitivity of the IACT $[10,11]$. The correction for GF effects required simulated $\gamma$-ray showers.

IACTs currently in operation offer improved imaging capabilities, i.e. better optical point spread function (PSF), pixel resolution and timing capabilities of the electronics, and are therefore more sensitive to GF effects than previous instruments.

In this paper we present results from dedicated MC studies of GF effects on the imaging technique. The studies were carried out for the Major Atmospheric Gamma-ray Imaging Cherenkov (MAGIC) telescope [12,13], which is located on the Canary Island of La Palma at the Roque de los Muchachos Observatory at $2200 \mathrm{~m}$ altitude $\left(28.45^{\circ} \mathrm{N}, 17.54^{\circ} \mathrm{W}\right)$.

\section{The MAGIC telescope}

The $17 \mathrm{~m}$ diameter MAGIC telescope is currently the largest single dish IACT in operation. The imaging camera in the focal plane of the tessellated parabolic reflector consists of 577 photomultiplier tubes (PMTs), all of which are arranged in a hexagonal configuration. The inner part of the camera is equipped with 397 PMTs of a diameter of $0.1^{\circ}$ whereas the outer part is equipped with larger PMTs of a diameter of $0.2^{\circ}$. The reflector has a focal length of $17 \mathrm{~m}$. The field of view of the camera is $3.5^{\circ}$ and the angular resolution for $\gamma$-rays is about $0.1^{\circ}$, depending on the energy. The telescope is in continuous operation since summer 2004. It allows for a detection of a $\gamma$-ray source with an absolute intensity of $\sim 2 \%$ of the Crab nebula and similar energy spectrum within $50 \mathrm{~h}$ at energies $>200 \mathrm{GeV}$ on a significance level of five standard deviations.

MAGIC is currently being upgraded through the addition of a twin telescope to achieve an improved sensitivity and a lower energy threshold [14]. Further technical details and information on the performance of the instrument can be found elsewhere [15].

\section{The GF at the MAGIC site}

The component of the GF normal to the shower axis is relevant for the east-west separation of electrons and positrons during the shower development. For this study the telescope optical axis has always been set parallel to the direction of the primary $\gamma$-ray.

Fig. 1(a) shows the absolute value of the GF component $\left|\vec{B}_{\perp}\right|$ normal to the direction of the EAS versus azimuth $(\mathrm{Az})$ angle and zenith angle (ZA) for the MAGIC telescope site. The value was determined for $10 \mathrm{~km}$ a.s.l. according to the epoch 2005 International Geomagnetic Reference Field (IGRF) model [17]. The MAGIC telescope is focused to a distance of $10 \mathrm{~km}$ a.s.l., which is the most likely location of the shower maximum for $100 \mathrm{GeV} \gamma$-ray-induced EAS at small ZAs. The trajectory of the strongest source of steady VHE $\gamma$-ray emission in the Galaxy, the Crab nebula, is indicated. As the magnetic field lines at La Palma are tilted by $\sim 7^{\circ}$ westwards with respect to the meridian [17] the trajectory is asymmetric with respect to $180^{\circ} \mathrm{Az}$ angle.

For La Palma, the minimum influence of the GF is expected to occur for EAS developing in direction of the magnetic north at $\mathrm{ZA}=\left(90^{\circ}-I\right) \approx 51^{\circ}$ and $\mathrm{Az}=0^{\circ}$, where the angle $\theta$ between the shower axis and the GF becomes smallest (see Fig. 1(b)), i.e. for EAS developing along the field lines. $I$ denotes the angle under which the GF lines dip into the Earth's surface, which is $\sim 39^{\circ}$ for La Palma [17]. Hence, the maximum influence is expected for EAS developing perpendicular to the direction of the GF lines, i.e. for $\mathrm{ZA} \approx 39^{\circ}$ and $\mathrm{Az}=180^{\circ}$.

The results from the MC studies on the GF effects presented here are specific to the MAGIC telescope because of the local GF strength and the relevant telescope parameters like the reflector area, camera pixelisation and the $\gamma$-ray PSF. a

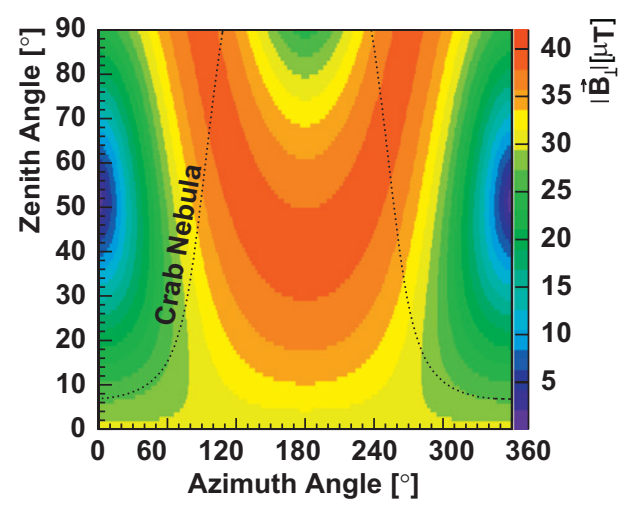

b

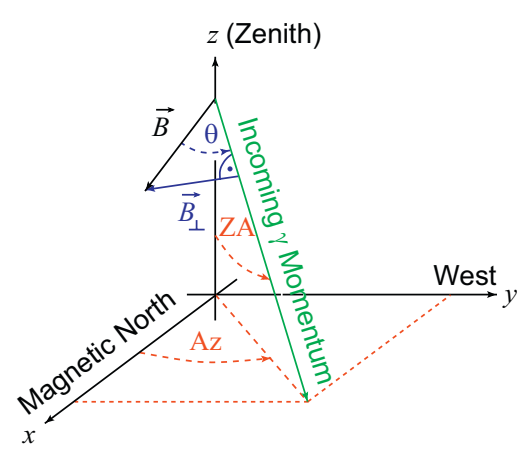

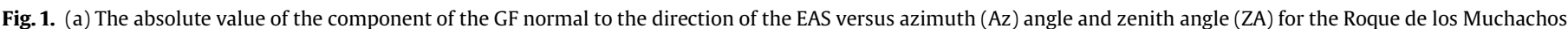

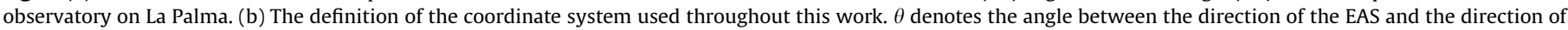

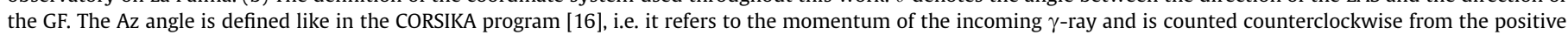
$x$-axis towards west. The telescope optical axis has always been set parallel to the direction of the primary $\gamma$-ray. 


\section{Monte Carlo simulations}

The production of Monte Carlo (MC) data for MAGIC involves three steps [18]:

1. The CORSIKA (COsmic Ray SImulations for KAscade) MC program (version 6.019) [16] is used to simulate the development of $\gamma$-ray and hadron-induced EAS and the production of Cherenkov light for a given set of input parameters, like the primary $\gamma$-ray or hadron energy, height above sea level, the magnitude and direction of the GF, and so on. The GF components are set to the values for the location of the MAGIC telescope (La Palma, $28.8^{\circ} \mathrm{N} 17.9^{\circ} \mathrm{W}$ ) which are provided by the IGRF model [17]. The $x$-axis of the Cartesian CORSIKA reference frame is aligned with the magnetic north pole and the $y$-axis points to the west. The $\mathrm{Az}$ angle is counted counterclockwise from the positive $x$-axis and refers to the direction of the primary $\gamma$-ray (Fig. 1(b)). Furthermore, the so-called US standard atmosphere is used as a model for the Earth's atmosphere.

2. The binary output of CORSIKA, containing information on the Cherenkov photon direction and its position on ground, is processed with a dedicated Reflector program, which does the ray-tracing of the Cherenkov photons. To be able to adapt to different conditions without being forced to rerun CORSIKA, atmospheric absorption and scattering of Cherenkov photons as well as mirror condition is taken into account at this stage.

3. Finally, the output of the Reflector program is processed by the Camera program simulating the entire readout chain, i.e. PMT response, trigger and data acquisition system including electronic noise. Normally, a compact next-neighbour coincidence is required, i.e. at least four neighbouring pixels are required to trigger, and, if any of the pixels is taken out of the group, the remaining pixels are still neighbours. To adapt the MC data to the optical performance of the telescope, the simulation of the optical PSF can be tuned at this stage. The calibration and the image parameter calculation (Hillas analysis [19]) is done using the MAGIC Analysis and Reconstruction Software (MARS) [20].

\subsection{Dedicated MC production}

For the present studies only $\gamma$-rays were simulated. The MC data were produced following for most instances the standard MC production of the MAGIC telescope as described beforehand. All events were simulated as originating from a point source. By definition, the telescope optical axis is always parallel to the direction of the primary $\gamma$-ray. The impact parameter (IP) is defined as the distance from the centre of the telescope mirror to the shower axis, which has the same direction as the primary $\gamma$-ray.

In contrast to the production of standard MC data, where the EAS core location is randomly placed somewhere in a circle on the plane perpendicular to the direction of the EAS (to estimate the telescope effective collection area), the EAS for this study were simulated for fixed core locations and all Cherenkov photons arriving in a circle of $200 \mathrm{~m}$ radius were kept. This procedure reduces computing time because each CORSIKA event can be used multiple times by placing the telescope (at the level of the Reflector program) somewhere into the circle within which Cherenkov photons are kept (Fig. 2(a)). Besides, this approach allows to study the influence of the GF on the shower images in great detail. For technical reasons the telescope was placed on equidistant points concentrically to the EAS core location. By the choice of this setup, the IP varies like $r \sqrt{\cos ^{2}(\mathrm{Az}-\varphi)\left(\cos ^{2}(\mathrm{ZA})-1\right)+1}$ (Fig. 2(b)), where $r$ is the distance between the EAS core location and the telescope position.

The energy of the primary $\gamma$-ray was for different samples set to $30,50,70,120,170,300,450$ or $1 \mathrm{TeV}$, respectively. The ZA was varied between $0^{\circ}$ and $60^{\circ}$ in steps of $20^{\circ}$, and the $A z$ angle between $0^{\circ}$ and $180^{\circ}$ in steps of $30^{\circ}$, as the absolute value of the GF component normal to the EAS direction is symmetric in the $\mathrm{Az}$ angle (Fig. 1(a)). Hence, the maximum value for the angle $\theta$ achieved with the simulated telescope orientations is $87^{\circ}$. The choice of discrete values for the $\gamma$-ray energy allows to investigate the energy dispersion of the showers due to the GF. The distance $r$ between the telescope position and the EAS core location (impact point of the primary $\gamma$-ray on the ground) was varied between 20 and $180 \mathrm{~m}$ in steps of $20 \mathrm{~m}$ and the angle $\varphi$ (Fig. 2(a)) between $0^{\circ}$ and $330^{\circ}$ in steps of $30^{\circ}$, resulting in 108 configurations.

About $10^{5}$ events were simulated for each $\gamma$-ray energy, ZA and $\mathrm{Az}$ angle. As a reference, MC data were also produced without GF. To be as realistic as possible the MC simulations include the effects of photons from the diffuse night sky background of $1.75 \times 10^{12} \mathrm{ph} \mathrm{m}^{-2} \mathrm{~s}^{-1} \mathrm{sr}^{-1}$ at the MAGIC site [21] as well as electronic noise. a

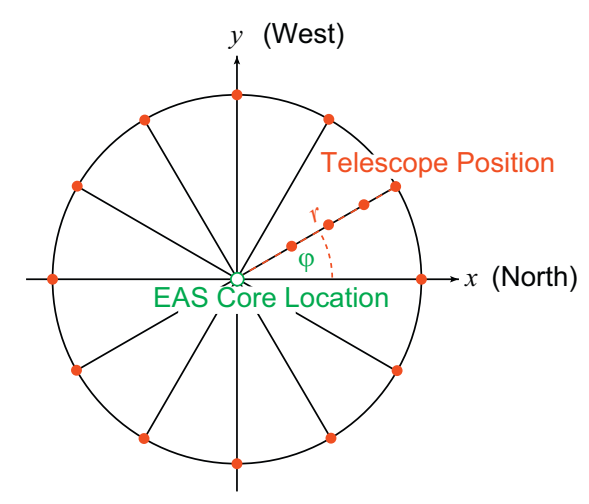

b

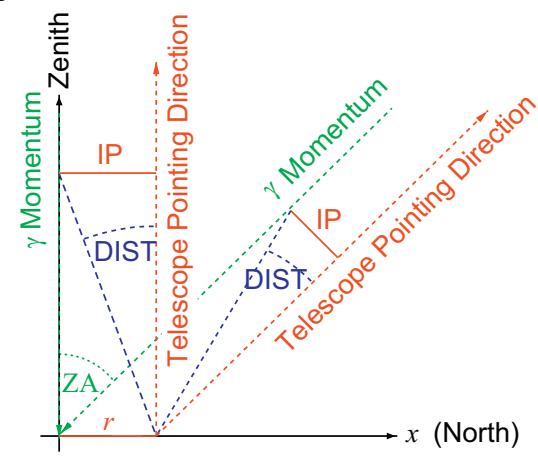

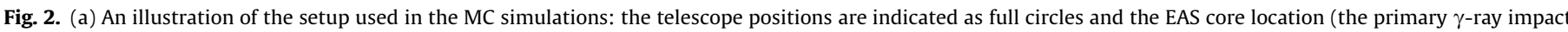

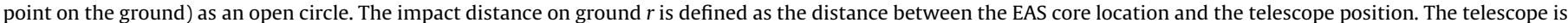

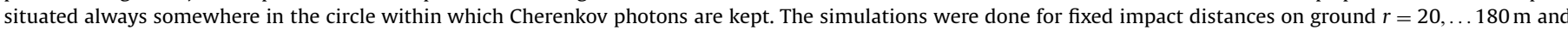

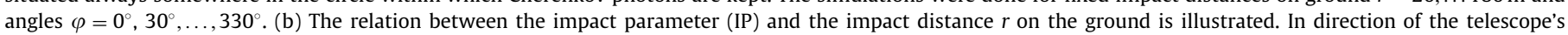
inclination the IP equals $r \cos (\mathrm{ZA})$. 


\section{Image analysis}

The MC-generated $\gamma$-ray showers were analysed using the standard MAGIC software MARS [20]. Before parameterisation of the shower images a tail-cut image cleaning was applied (Fig. 3(a)) [15]. The image cleaning requires the signals to be above a certain level. For the MC studies presented here the minimum required pixel content was seven photoelectrons (phe) for so-called core pixels and four phe for boundary pixels.

Shower images from $\gamma$-ray showers processed with the image cleaning are narrow and point towards the source position in the field of view. To a first approximation the shower images are elliptical and can be described by so-called Hillas parameters [19]. Detailed reviews on the imaging technique can be found elsewhere [7,22]. Some of the image parameters used for these studies are illustrated in Fig. 3(b). The parameters WIDTH and LENGTH characterise the lateral and longitudinal spread of the shower images (minor and major axes of the so-called Hillas ellipse). Both parameters are very important since they allow a powerful discrimination of $\gamma$-ray images against hadron-induced images. The parameters DIST and ALPHA are related to the position and orientation of shower images in the camera. The parameter DIST is directly related to the IP of the primary $\gamma$-ray (Fig. 2(b)). The image parameter ALPHA is commonly used by standalone IACTs to extract the $\gamma$-ray signal. ALPHA denotes the angle between the major axis of the shower image and the vector connecting its centre of gravity with the source position in the camera plane (camera centre). It provides a very powerful discrimination between $\gamma$-ray images from a point-like source and any isotropic background (mainly due to hadrons), i.e. orientation discrimination. The $\gamma$-ray signal from a VHE $\gamma$-ray source under study appears as an excess at small values in the ALPHA parameter distribution.

Another viable image parameter is the so-called SIZE, which corresponds to the total integrated light of a shower image after treatment with the image cleaning procedure. It is therefore an estimate for the primary $\gamma$-ray energy.

The so-called DISP method [23,24] allows to reconstruct the arrival direction of $\gamma$-ray candidates making use of the shape of a shower image. The DISP parameter is determined according to the formula

$\mathrm{DISP}=c_{1}(\mathrm{SIZE})+c_{2}(\mathrm{SIZE}) \cdot \frac{\mathrm{WIDTH}}{\mathrm{LENGTH}}$.

a

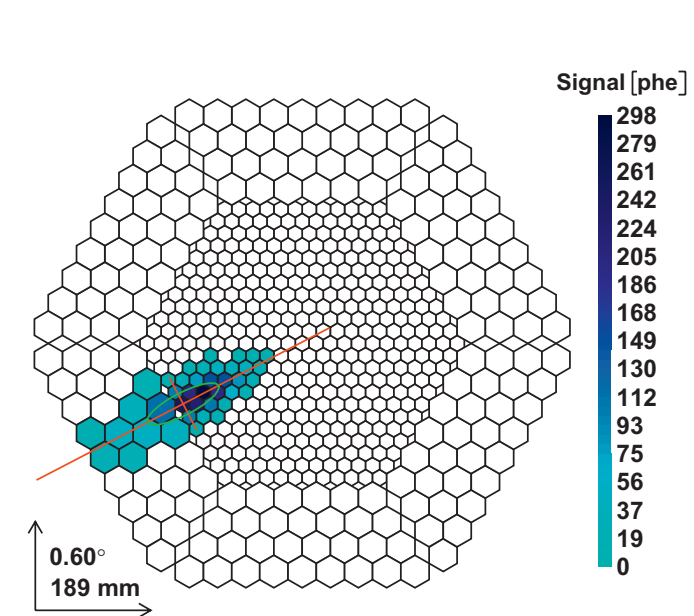

Therein $c_{1}$ and $c_{2}$ are second-order polynomials optimised on MC simulated $\gamma$-ray showers [25]:

$c_{1}=+1.163^{\circ}+0.542^{\circ}\left(\log _{10}(\mathrm{SIZE})-2\right)-0.672^{\circ}\left(\log _{10}(\mathrm{SIZE})-2\right)^{2}$

$c_{2}=-0.265^{\circ}-2.905^{\circ}\left(\log _{10}(\mathrm{SIZE})-2\right)+2.220^{\circ}\left(\log _{10}(\mathrm{SIZE})-2\right)^{2}$.

In case of a single telescope the DISP method provides two possible solutions for the source position. To overcome this ambiguity the asymmetry of the shower image along the major image axis, which is related to the longitudinal development of an EAS in the atmosphere, is used to reconstruct the true source position. However, owing to false head-tail assignment the percentage of correctly reconstructed events is typically limited to $\sim 80 \%$, depending on the $\gamma$-ray energy [25]. The outcome of the DISP analysis is usually displayed in terms of a sky map of arrival directions. Because the DISP parameter depends on both the eccentricity WIDTH/LENGTH and on the orientation of the shower images the influence of the GF on the shower development is expected to degrade also the DISP method.

In this paper, we restrict ourselves to selected but representative results from the MC study.

\section{Results and discussion}

\subsection{GF effects on shape and orientation of $\gamma$-ray shower images}

The orientation of the $\gamma$-ray images from a source under study is used for the suppression of the isotropic hadronic background. It is accordingly important to study how the GF influences the orientation of shower images. Fig. 4 shows shower images (Hillas ellipses) in the telescope camera for $450 \mathrm{GeV} \gamma$-rays, $100 \mathrm{~m} \mathrm{IP}$, $\mathrm{ZA}=40^{\circ}$, and different $\mathrm{Az}$ angles between $0^{\circ}$ and $180^{\circ}$. The ellipses drawn with solid lines were obtained for enabled GF and the ones drawn with dashed lines for disabled GF in the MC simulation. For each angle $\varphi=0^{\circ}, 30^{\circ}, \ldots, 330^{\circ}$ the size, the position and the orientation of the ellipse in the camera was determined by taking the mean values from the corresponding Hillas parameter distribution. The ellipses are superimposed on the projection of the CORSIKA coordinate system, whose $x$-axis is aligned with the magnetic north. The direction of the GF component normal to the direction of the EAS is indicated in the lower right part of the figures.

b

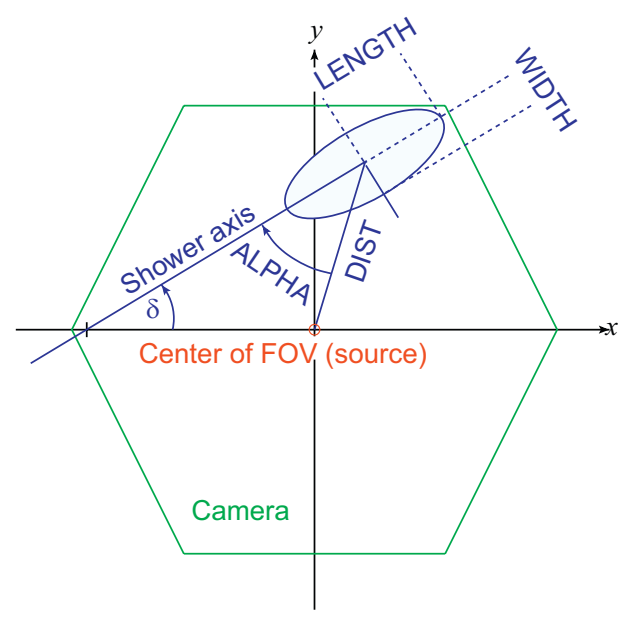

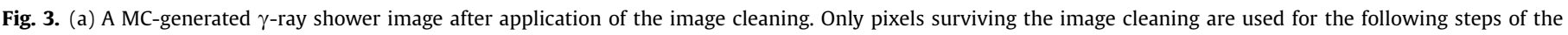

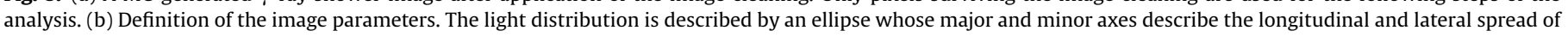
the Cherenkov light distribution. 
a

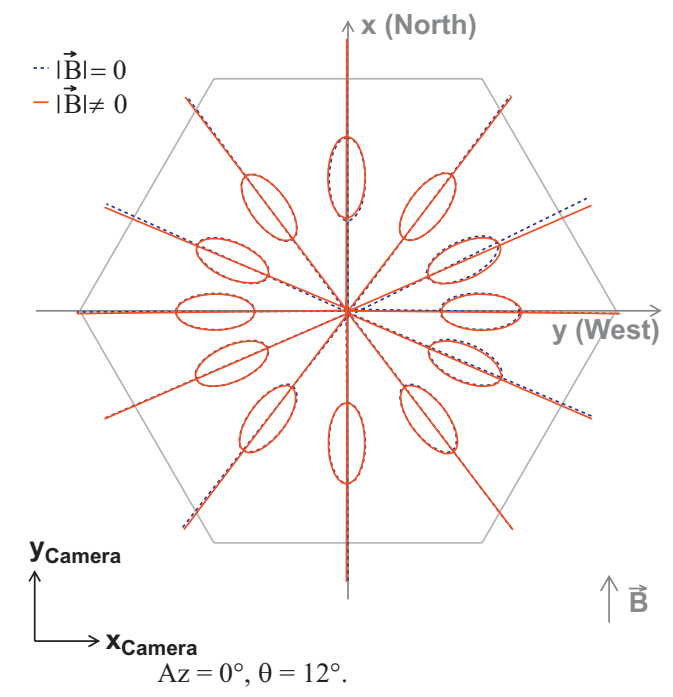

b

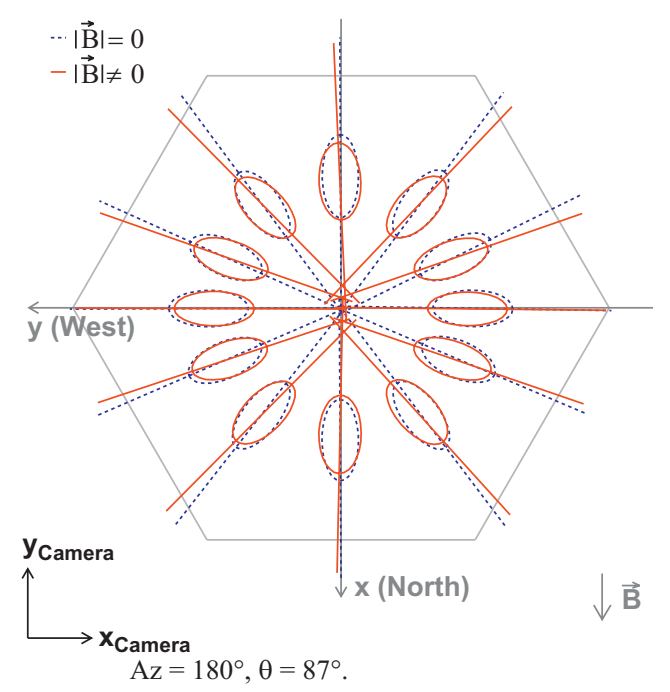

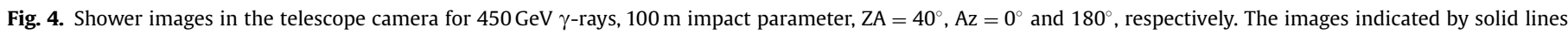

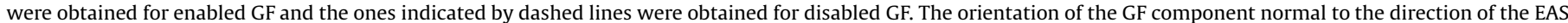
(telescope pointing direction) is indicated in the lower right part of the figures (see text for more details).

As can be seen the average orientation is preserved only for shower images oriented either parallel or normal to the projected direction of the GF in the camera, which is by definition the GF component normal to the direction of the EAS (telescope pointing direction). Shower images situated at intermediate angles are systematically rotated away from the projected direction of the GF. The sideways spread of the images result in a systematic rotation away from the camera centre (source position). This was also reported in Ref. [10]. The magnitude of the rotation depends not only on the angle $\theta$ between the axis of the EAS and the direction of the GF but mainly on the core position of the EAS with respect to the telescope, which is given by the angle $\varphi$.

By comparing the orientations for images generated with disabled GF to the ones for enabled GF it is possible to determine the rotation angle. Fig. 5 shows the rotation angle of $\gamma$-ray images versus energy for IPs of 40 and $120 \mathrm{~m}$. The figures show that the rotation angle depends on the IP and on the $\gamma$-ray energy. However, the average rotation angle for images oriented either parallel or normal to the direction of the GF in the camera is zero (full square and full triangle down data points). Images oriented at intermediate angles are systematically rotated. For $Z A=0^{\circ}$, $\mathrm{Az}=0^{\circ}$ and $40 \mathrm{~m} \mathrm{IP}$ (Fig. 5(a)) and for $\mathrm{ZA}=40^{\circ}, \mathrm{Az}=180^{\circ}$ (Fig. 5(c) and (d)), which is the most unfavourable telescope pointing direction, the rotation angle is maximal for $\gamma$-ray energies around $450 \mathrm{GeV}$.

Fig. 6 illustrates the dependency of the rotation angle on the IP. Small IPs correspond to images with low eccentricity WIDTH/LENGTH, which may be rotated through a large angle. Fig. 6(b) also shows that the direction of the rotation depends on the IP. The shower images are not always rotated away from the direction of the GF in the camera but can even be rotated towards it. Consequently, the correction of observational data for GF effects by de-rotating the shower images must take into account the energy dependence of the rotation angle and its dependence on the IP (DIST). For the most unfavourable telescope pointing direction and for small IPs even $1 \mathrm{TeV}$ shower images are rotated through a large angle (Fig. 6(f)). The influence of the GF on the shape of the $\gamma$-ray shower images also depends on the primary $\gamma$-ray energy, the IP and the orientation of the EAS relative to the direction of the GF. Fig. 7 shows the average WIDTH and LENGTH of $\gamma$-ray images versus energy for $120 \mathrm{~m} \mathrm{IP,} \mathrm{ZA}=40^{\circ}$, and $0^{\circ}$ as well as $A z=180^{\circ}$. For the most unfavourable telescope pointing direction, i.e. $\mathrm{ZA}=40^{\circ}$ and $\mathrm{Az}=180^{\circ}\left(\theta=87^{\circ}\right)$ significant GF effects on the image parameter WIDTH occur for $\gamma$-ray energies above $\sim 100 \mathrm{GeV}$ (Fig. 7(b)). The influence of the GF on the image parameter WIDTH is considerably larger than on LENGTH. Due to the influence of the GF the average WIDTH is increased for images where the connecting line between the EAS core location on ground and the telescope position is parallel to the magnetic north-south direction (telescope situated on the $x$-axis, full and open circle data points). Images aligned with the direction of the GF in the camera are horizontally stretched compared to the situation of disabled GF in the MC, whereas images oriented normal to the direction of the GF have a smaller WIDTH and are thus elongated due to the influence of the GF (telescope situated on the $y$-axis, full and open square data points).

\subsection{GF effects on the image parameter ALPHA}

In the preceding section it was shown that the GF can strongly alter the average shape and orientation of $\gamma$-ray shower images in the camera. Even though the orientation of shower images and the image parameter ALPHA are correlated it is important to investigate the influence of the GF on the image parameter providing the most powerful discrimination between $\gamma$-rays from a point-like source and unwanted isotropic background (mainly hadrons).

Figs. 8-10 show the ALPHA distributions (normalised to the number of entries) for $\gamma$-ray energies of 50,450 and $1 \mathrm{TeV}$. Showers recorded at 40 and $120 \mathrm{~m}$ IP were considered together with the different possible configurations: the distributions indicated by dash-dotted lines correspond to $\varphi=0^{\circ}$ and $180^{\circ}$ (as defined in Fig. 2(a)), where the connecting line between the shower axis and the telescope optical axis is parallel to the north-south direction. This configuration corresponds to shower images which are oriented parallel to the direction of the GF in the camera. The distributions indicated by solid lines were obtained for $\varphi=90^{\circ}$ and $270^{\circ}$, where the connecting line between the shower axis and the telescope optical axis is parallel to the east-west direction. In this case the shower images are on average not rotated and oriented normal to the direction of the GF in the 
a

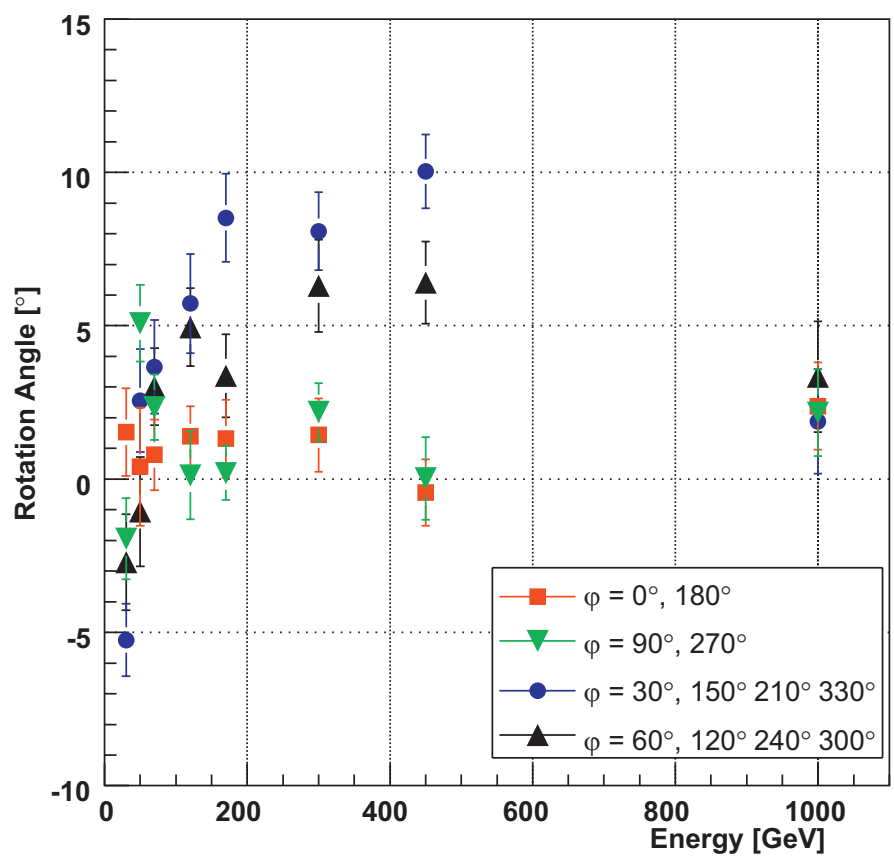

$\mathrm{Az}=0^{\circ}, \mathrm{ZA}=0^{\circ}, \mathrm{IP}=40 \mathrm{~m}, \theta=52^{\circ}$.

C

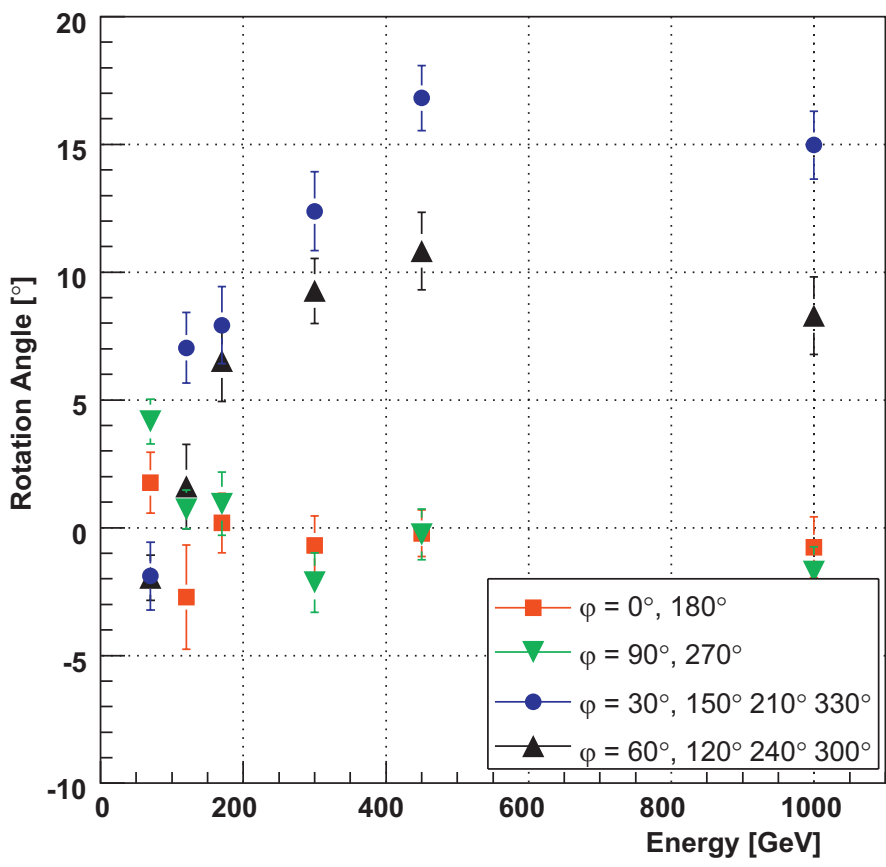

b

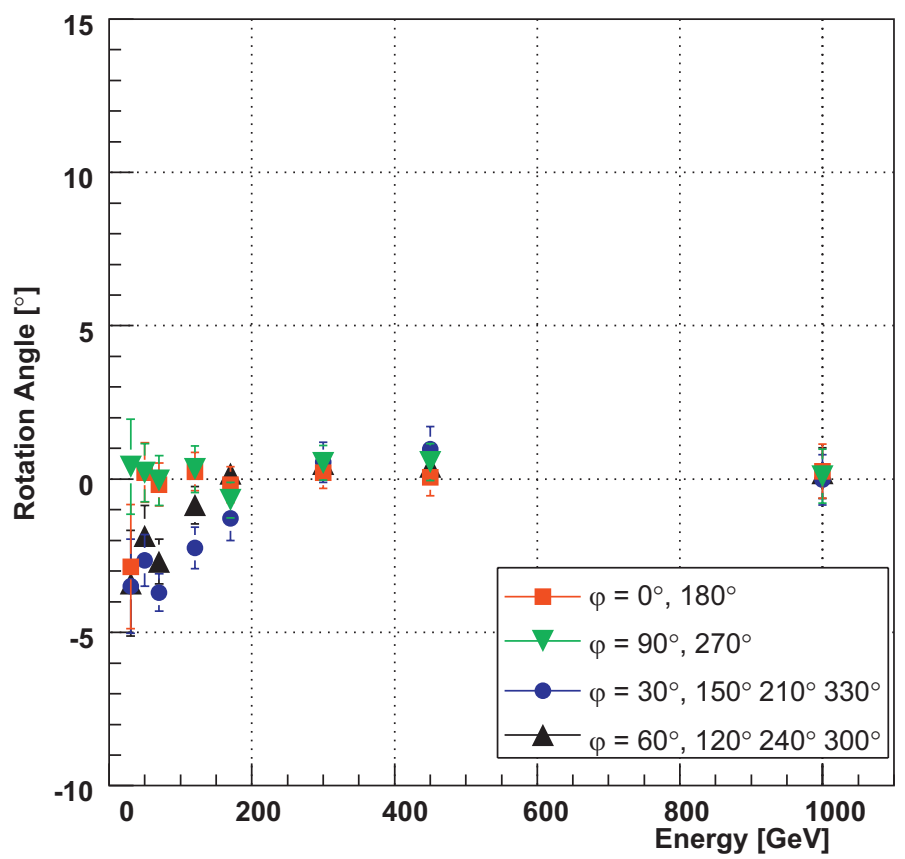

$\mathrm{Az}=0^{\circ}, \mathrm{ZA}=0^{\circ}, \mathrm{IP}=120 \mathrm{~m}, \theta=52^{\circ}$.

d

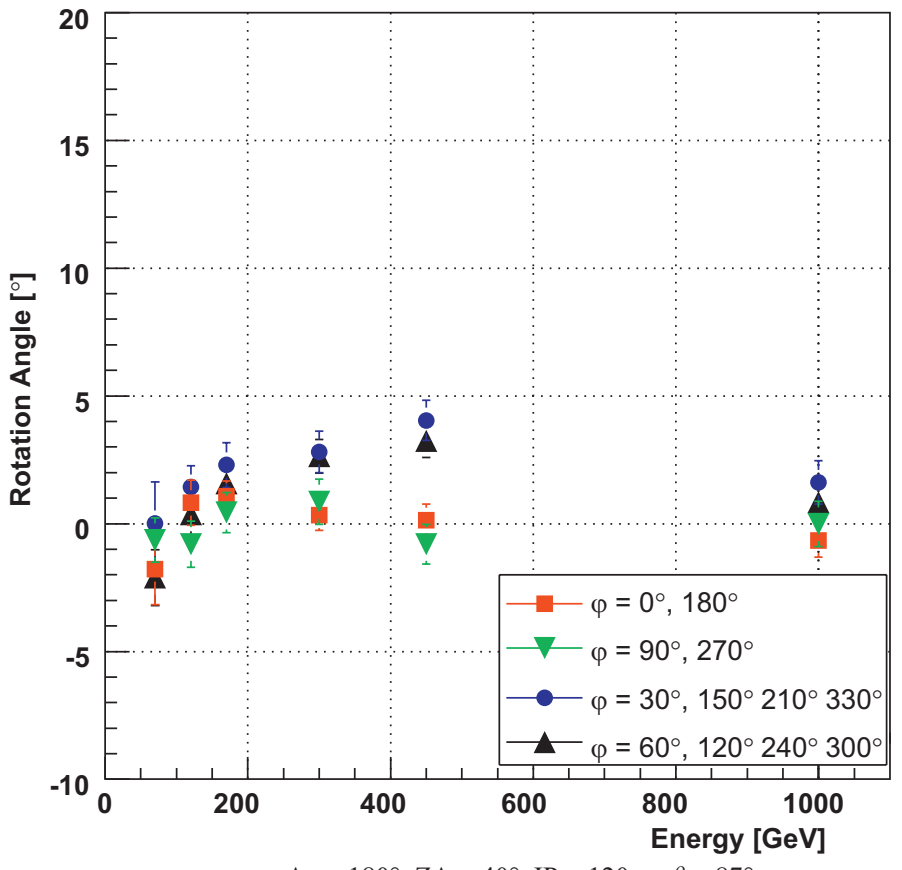

$\mathrm{Az}=180^{\circ}, \mathrm{ZA}=40^{\circ}, \mathrm{IP} \approx 120 \mathrm{~m}, \theta=87^{\circ}$.

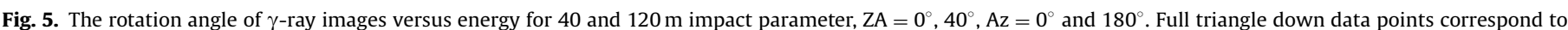

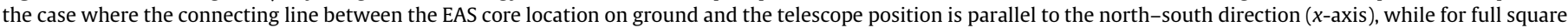
data points the telescope is situated on the $y$-axis. Full triangle up and full circle data points correspond to intermediate telescope positions.

camera. The ALPHA distributions indicated by dashed lines belong to intermediate telescope positions $\varphi=30^{\circ}, 60^{\circ}, 120^{\circ}, 150^{\circ}, 210^{\circ}$, $240^{\circ}, 300^{\circ}$ and $330^{\circ}$. The corresponding ALPHA distributions obtained without GF in the MC simulation are also plotted (dotted lines). The percentage of events with $|\mathrm{ALPHA}| \leqslant 9^{\circ}$ is given in the legend. The cut is indicated by the vertical dotted line.

It can be seen that for configurations where the connecting line between the telescope and the shower axis is parallel to the north-south direction (parallel to the direction of the GF) the corresponding ALPHA distributions (red histograms) can be significantly broadened although the corresponding shower images are on average not rotated. However, the ALPHA distribution for the opposite configuration (connecting line between the telescope and the shower axis parallel to the east-west direction) are stronger peaked at low values due to the influence of the GF (compare Fig. 9(a) and (b)). The remaining 
a

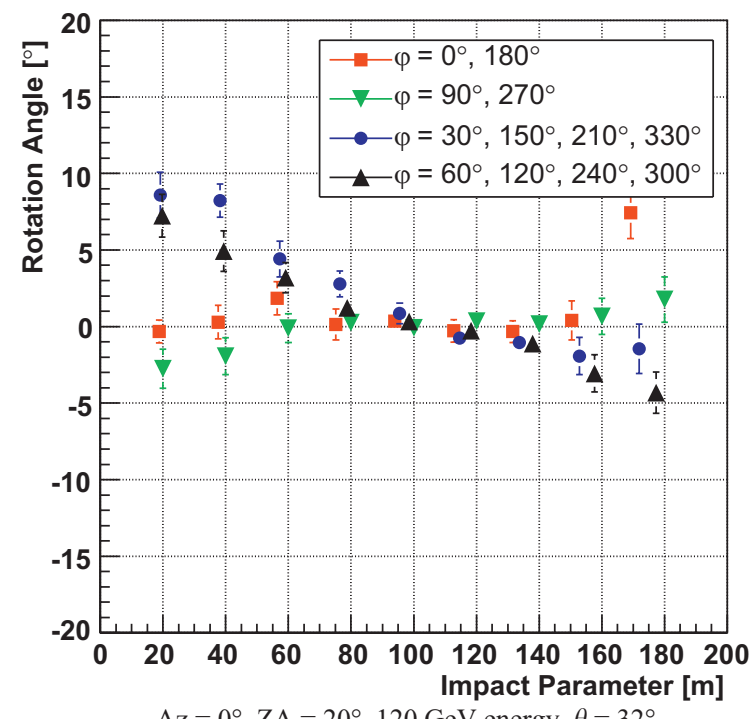

C

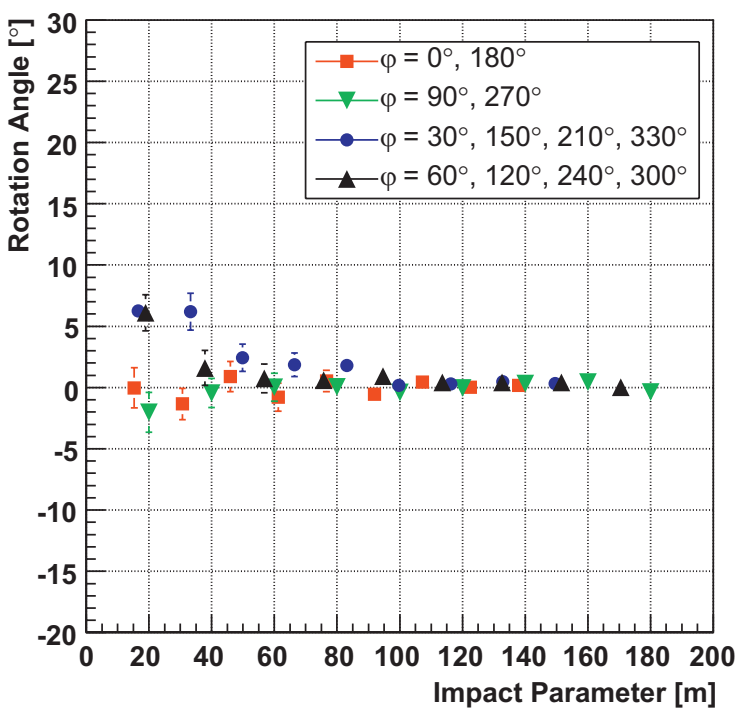

$\mathrm{Az}=0^{\circ}, \mathrm{ZA}=40^{\circ}, 450 \mathrm{GeV}$ energy, $\theta=12^{\circ}$.

e

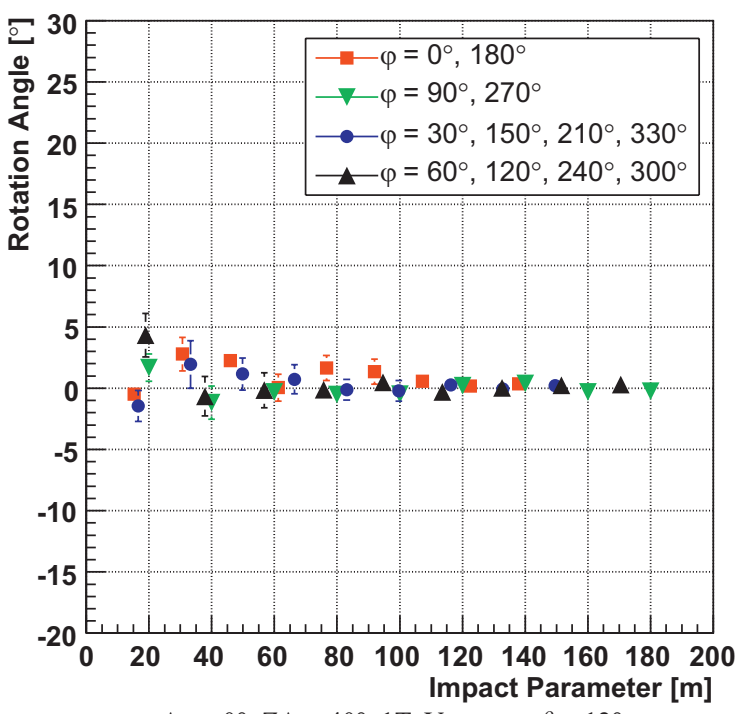

b

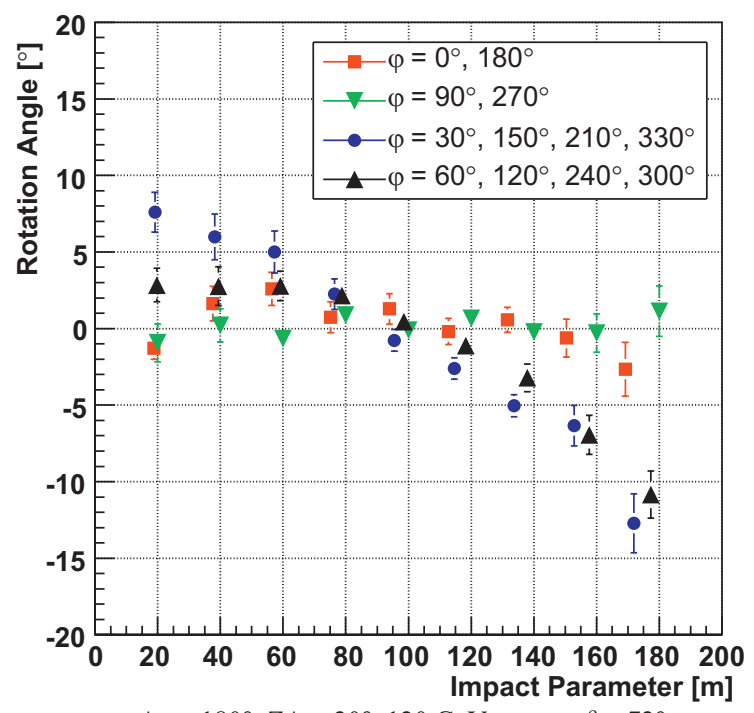

d

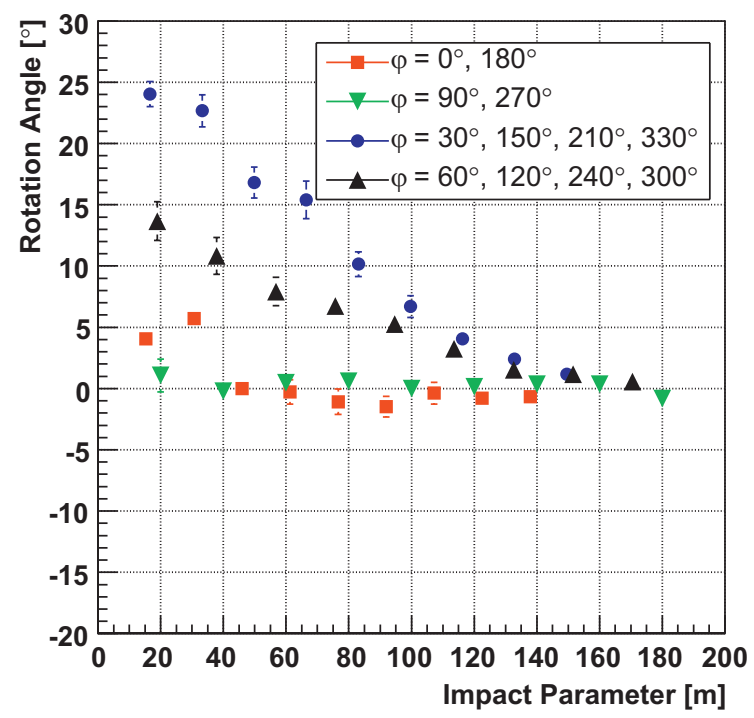

$\mathrm{Az}=180^{\circ}, \mathrm{ZA}=40^{\circ}, 450 \mathrm{GeV}$ energy, $\theta=87^{\circ}$.

$f$

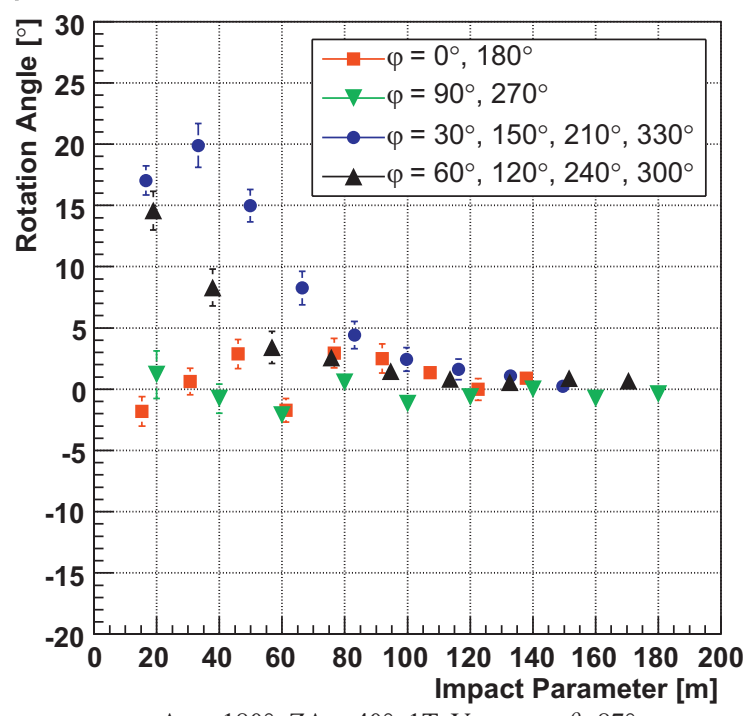

$\mathrm{Az}=180^{\circ}, \mathrm{ZA}=40^{\circ}, 1 \mathrm{TeV}$ energy, $\theta=87^{\circ}$.

Fig. 6. The rotation angle of $\gamma$-ray images versus impact parameter for $\mathrm{ZA}=20^{\circ}$ and $40^{\circ}, \mathrm{Az}=0^{\circ}$ and $180^{\circ}, 120 \mathrm{GeV}, 450 \mathrm{GeV}$ and $1 \mathrm{TeV}$ energy (see text for more details). 
a

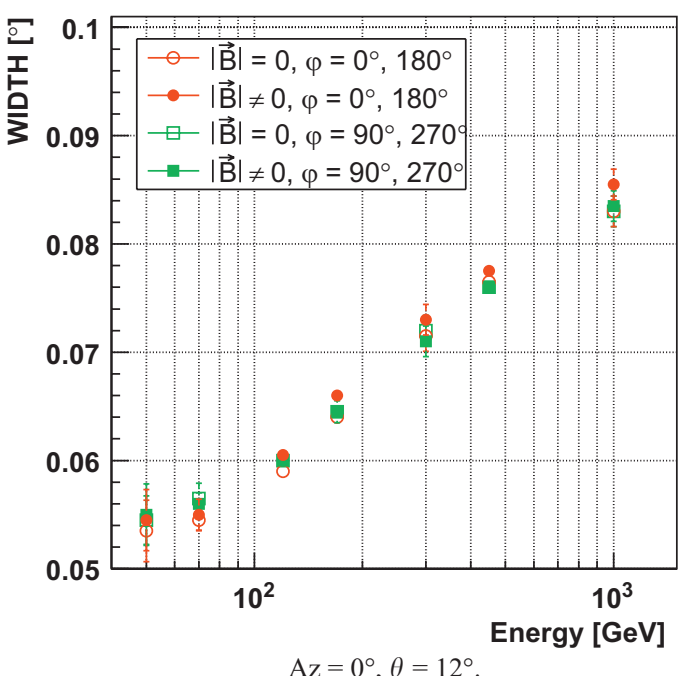

C

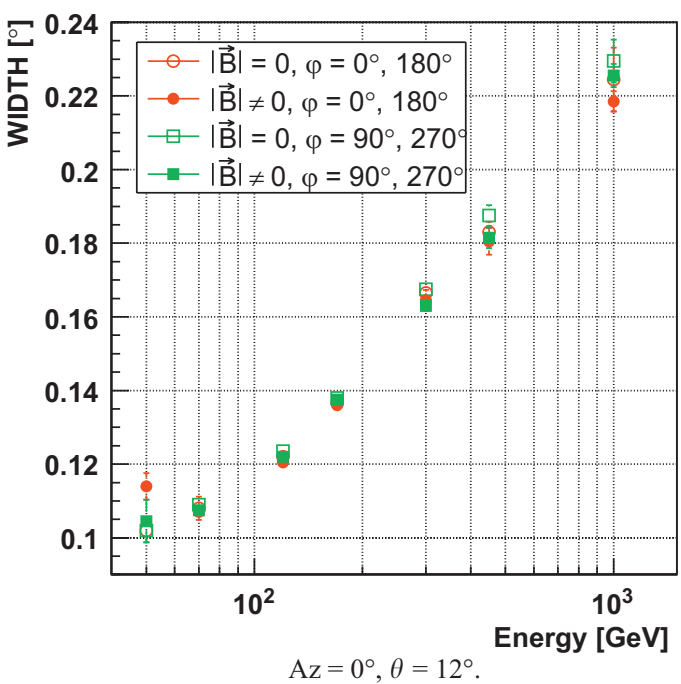

b

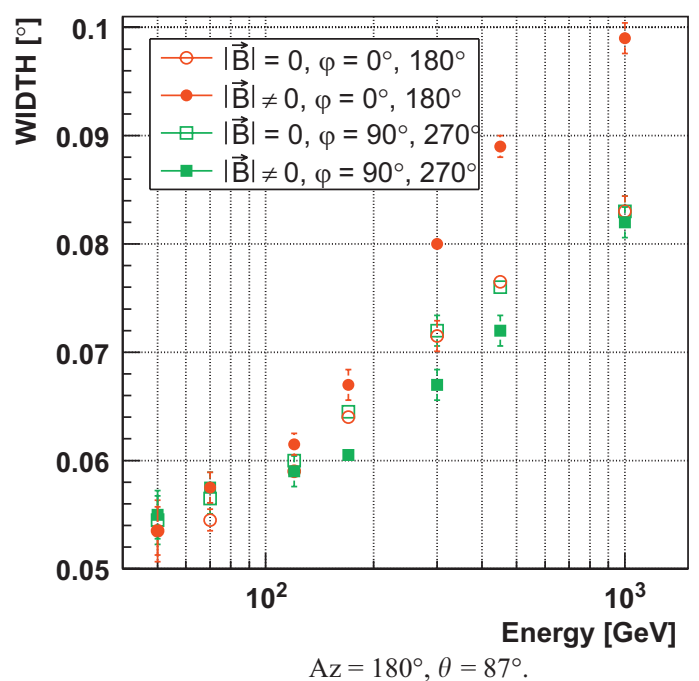

d

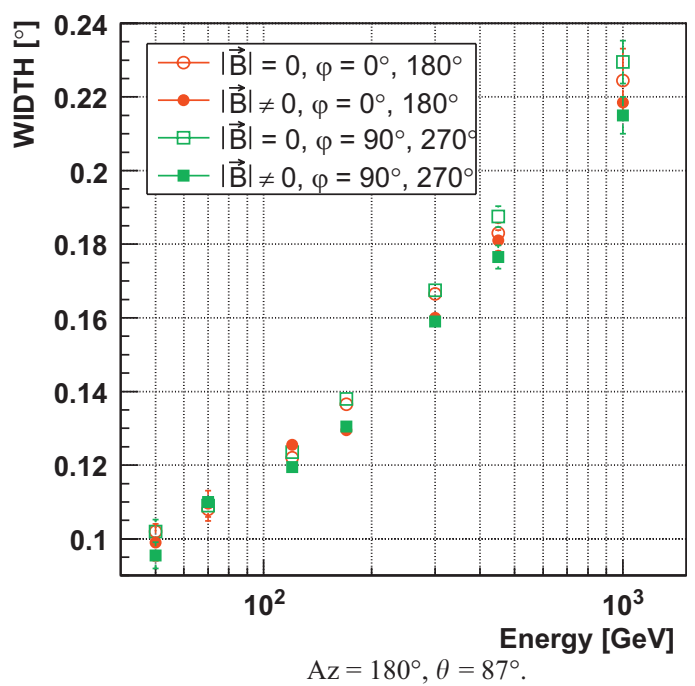

Fig. 7. The average WIDTH and LENGTH of $\gamma$-ray images versus energy for $120 \mathrm{~m}$ impact parameter, $\mathrm{ZA}=40^{\circ}, \mathrm{Az}=0^{\circ}$ and $180^{\circ}$ (see text for more details).

configurations always lead to broadened ALPHA distributions due to the rotation of the shower images with a preferential direction.

In conclusion it can be stated that the influence of the GF can significantly degrade the orientation discrimination of shower images. It is evident that for some configurations discussed above the $\gamma$-ray signal cannot be recovered by de-rotating the shower images.

Fig. 11 shows the ALPHA distributions for $450 \mathrm{GeV} \gamma$-rays, $\mathrm{ZA}=40^{\circ}, \mathrm{Az}=180^{\circ}$ and $40 \mathrm{~m}$ as well as $120 \mathrm{~m}$ IP. The ALPHA distributions obtained without GF (dotted line) are shown together with the distributions obtained for enabled GF (dashed line) and the ones obtained after de-rotation of the shower images (solid line). It is possible to correct for GF effects by de-rotating the shower images. However, if all telescope positions are taken into account $\left(\varphi=0^{\circ} \ldots 330^{\circ}\right)$ the improvement in terms of the percentage of events with $|A L P H A| \leqslant 9^{\circ}$ is less than $8 \%$ (Fig. 11(a) and (b)). Ignoring the most unfavourable configurations with respect to the influence of the GF $\left(\varphi \neq 0^{\circ}\right.$ and $\left.180^{\circ}\right)$ results in ALPHA distributions which are stronger peaked at small values (Fig. 11(c) and (d)). As expected, for the most unfavourable configurations $\left(\varphi=0^{\circ}\right.$ and $\left.180^{\circ}\right)$ the $\gamma$-ray signal cannot be recovered by de-rotation. The corresponding images are not rotated but their angular distribution is broadened (Fig. 11(e) and (f)).
Given that the energy, the rotation angle and the IP is well known in MC, the amount of recovered real $\gamma$-ray showers from observational data by de-rotation is expected to be lower. Both the energy and the IP have to be estimated and are thus known less precisely. To be efficient, the de-rotation of the shower images requires a precise knowledge of the IP and the information on the energy of the $\gamma$-ray candidates from observational data. Moreover, we focused on intermediate $\gamma$-ray energies where the rotation angle is large (Fig. 5(d)) and the spread of the ALPHA distribution is rather low. At lower energies than those considered here the recovery of the $\gamma$-ray signal is even less efficient [1]. This is also the case for $1 \mathrm{TeV} \gamma$-rays, where the ALPHA distribution is stronger peaked at small values and the rotation angle is smaller (Fig. 5). Because of the relatively poor knowledge of the IP in case of real shower images the improvement in sensitivity by de-rotation of the shower images is expected to be below $10 \%$.

\subsection{GF effects on the DISP-reconstructed $\gamma$-ray arrival direction}

A DISP analysis of the MC $\gamma$-ray shower images was performed to study the GF effects on the reconstructed arrival directions. 
a

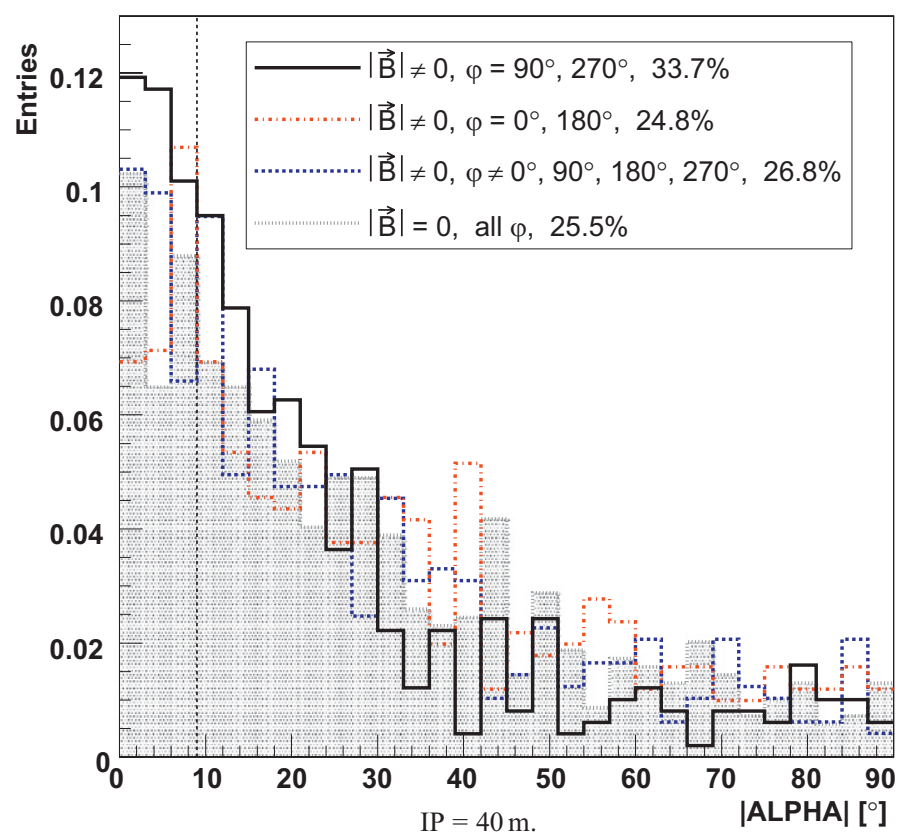

b

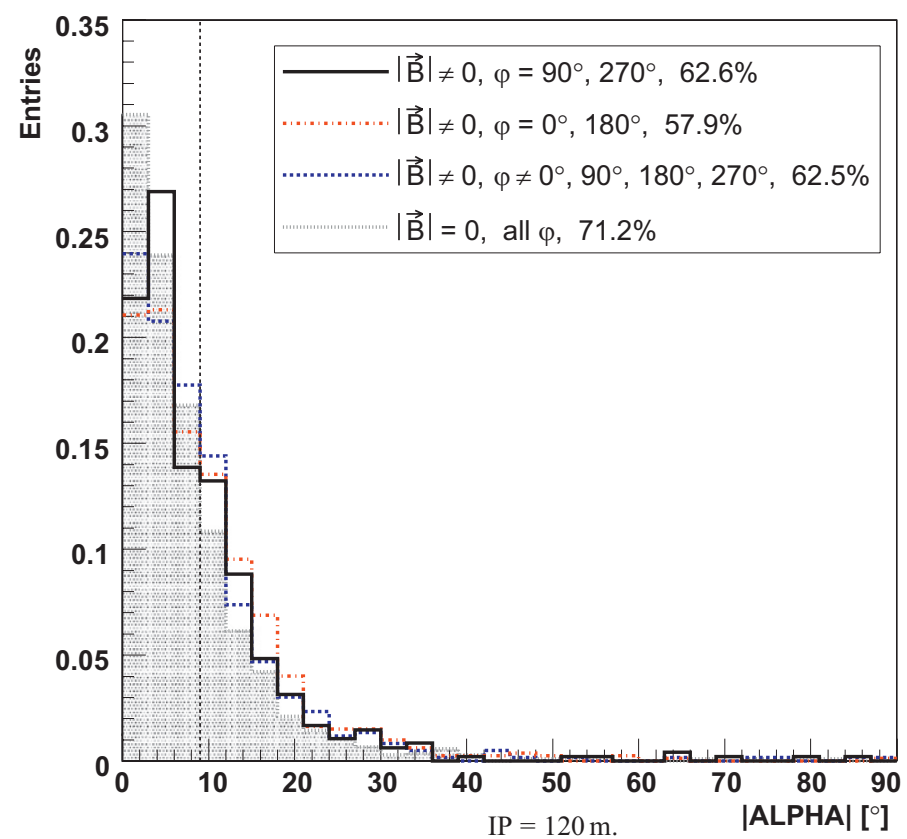

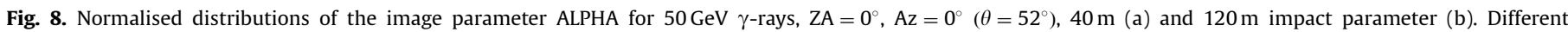

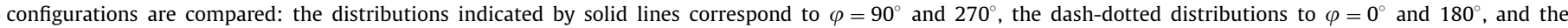

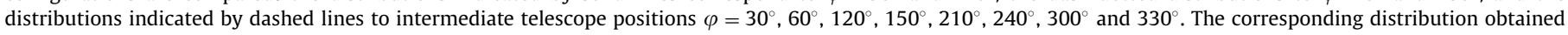
without GF in the MC simulation is also plotted (dotted line). The percentage of events with $|\mathrm{ALPHA}| \leqslant 9^{\circ}$ is given in the legend.

For the optimisation of the second-order polynomials in Eq. (1), dedicated MC $\gamma$-ray samples with continuous IP distribution between 0 and $500 \mathrm{~m}$ were produced. The MC samples were produced for the same $\gamma$-ray energies, ZAs and image cleaning levels as the MC data used for the preceding studies. The EAS core location was randomly placed somewhere in a circle on the plane perpendicular to the direction of the EAS. Also, the MC samples were produced without GF, thus only for $A z=0^{\circ}$. In this way the results from the DISP method obtained for different telescope pointing directions are comparable since the DISP polynomials themselves are not subject to GF effects.

Fig. 12 shows the DISP-reconstructed arrival directions for $450 \mathrm{GeV} \gamma$-rays, IPs between 60 and $140 \mathrm{~m}, \mathrm{ZA}=40^{\circ}, \mathrm{Az}=0^{\circ}$ and $180^{\circ}$. The projected direction of the GF is indicated in the lower right part of the figures, and the ellipticity of the distributions of DISP-reconstructed arrival directions is shown in the lower left part of the figures. The semi-minor and the semi-major axis of the ellipse correspond to the sigma of a Gaussian fit to the distributions using bands of $\Delta_{X, Y}= \pm 0.035^{\circ}$ parallel and perpendicular to the projected direction of the GF. The size of the bands was arbitrarily chosen, but it is not critical for the result. Within errors, the relative difference of the results obtained from the two orientations is independent of the size of the bands. The result from the Gaussian fit is also shown in the legend.

As can be seen from the figures the distributions appear to be significantly elongated perpendicular to the projected direction of the GF, while the peak of the DISP distribution is always centred at the nominal source position (camera centre). The extent of the elongation depends on the angle $\theta$ between the shower axis and the direction of the GF. The GF effects on the DISP method thus result in a degradation of the sky maps in a way that a point-like $\gamma$-ray source appears to be extended, i.e. the $\gamma$-ray PSF is degraded. The star-shaped appearance of the DISP distributions arises from events with wrong head-tail assignment. The false head-tail assignment cannot be attributed to GF effects since it occurs also for the favourable telescope pointing direction (Fig. 12(a)). This illustrates the basic limitation of a single telescope to properly reconstruct the true source position. In case of a single telescope the DISP method has to rely on the shower asymmetry along the major axis of the shower image (see Section 5).

Fig. 13 shows the lateral and longitudinal spread versus $\gamma$-ray energy for different orientations of the telescope. The spread is defined as the sigma of a Gaussian fit to the DISP distribution using bands of $\Delta_{X, Y}= \pm 0.035^{\circ}$ parallel and normal to the projected direction of the GF in the camera. IPs between 60 and $140 \mathrm{~m}$ were considered. The figures clearly show that, compared to the case of disabled GF, the spread of the DISP distribution increases significantly for an unfavourable telescope orientation (large angle $\theta$ ). The maximum spread occurs always perpendicular to the direction of the GF in the camera. Therefore, depending on the orientation of an EAS with respect to the telescope and the IP, the DISP-reconstructed incoming direction of the corresponding primary $\gamma$-ray has a large uncertainty.

\subsection{GF effects on the energy reconstruction of $\gamma$-ray images}

It was previously discussed that the influence of the GF on the shower development affects also the energy reconstruction and the trigger efficiency for $\gamma$-rays.

The east-west separation of electrons and positrons in EAS due to the GF modifies the Cherenkov distribution on the ground such that the reconstructed Cherenkov light, i.e. the integrated light content of the shower images is reduced for unfavourable shower orientations with respect to the direction of the GF.

Fig. 14 shows the average reconstructed light content of shower images together with the $\gamma$ efficiency versus $A z$ angle for $450 \mathrm{GeV}$ energy $\gamma$-rays and ZAs between $0^{\circ}$ and $180^{\circ}$. To compare equivalent configurations (see Fig. 2(a)) the telescope is always situated at angles $\varphi=\mathrm{Az}+90^{\circ}$ and the impact parameter 
a

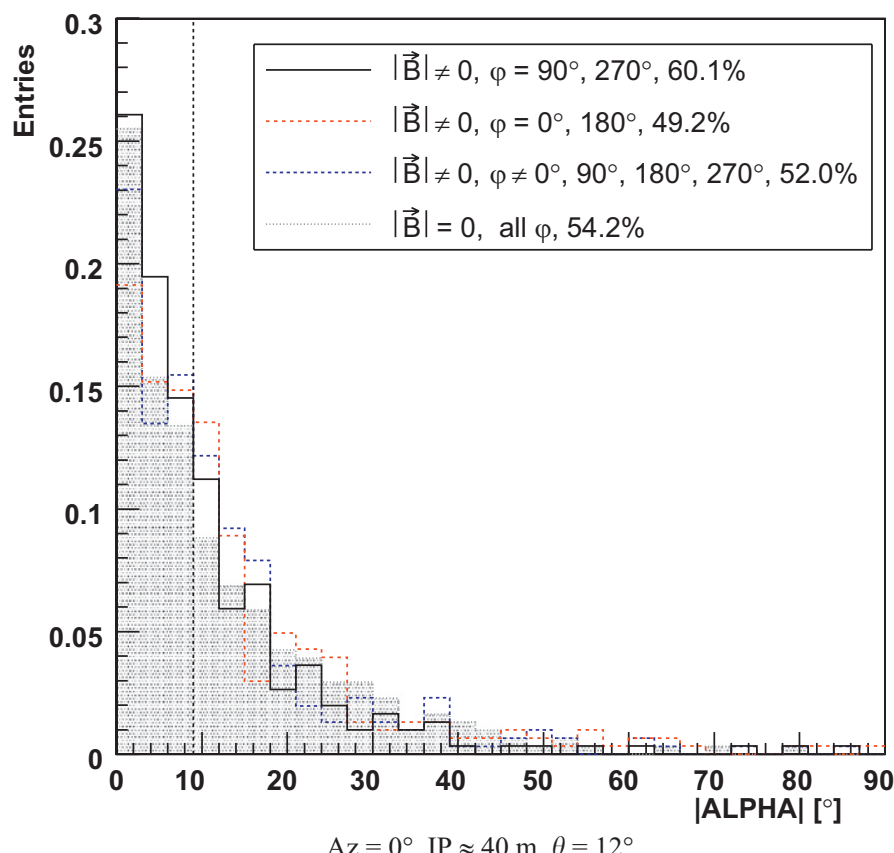

C

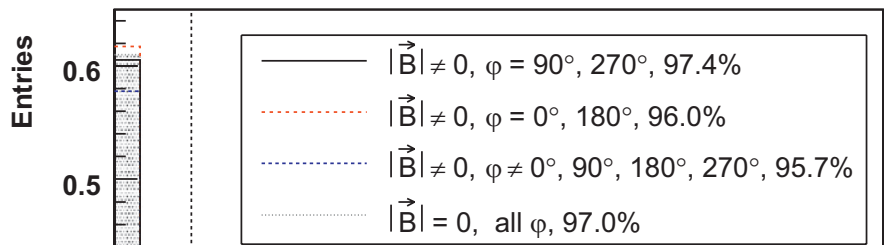

b

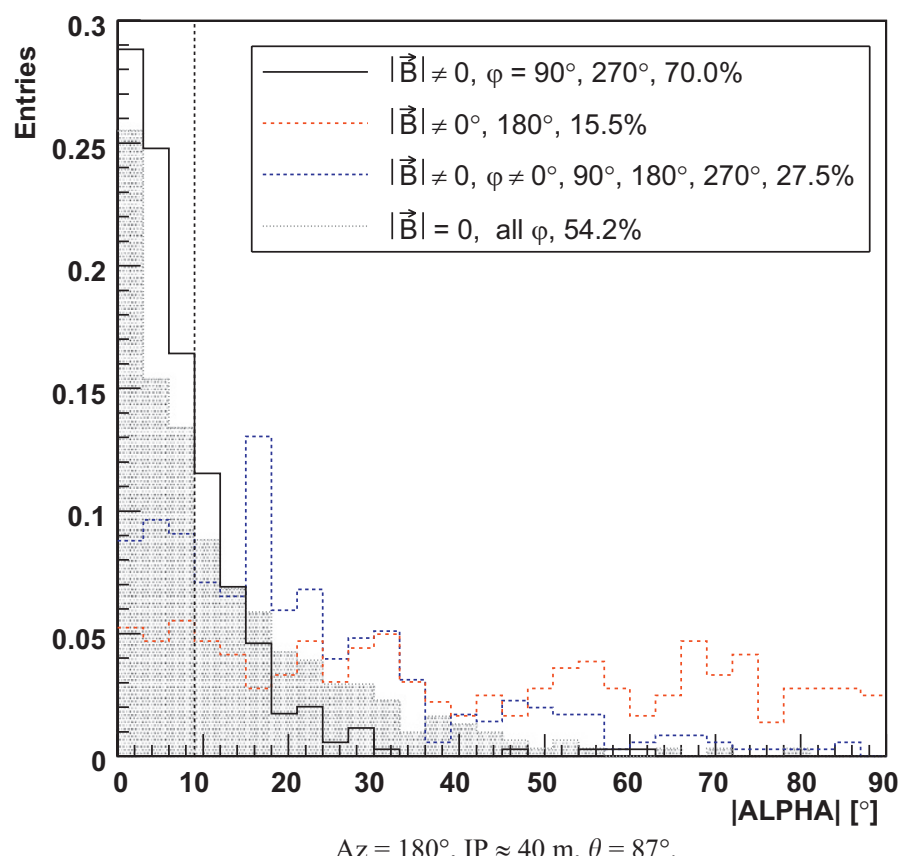

d

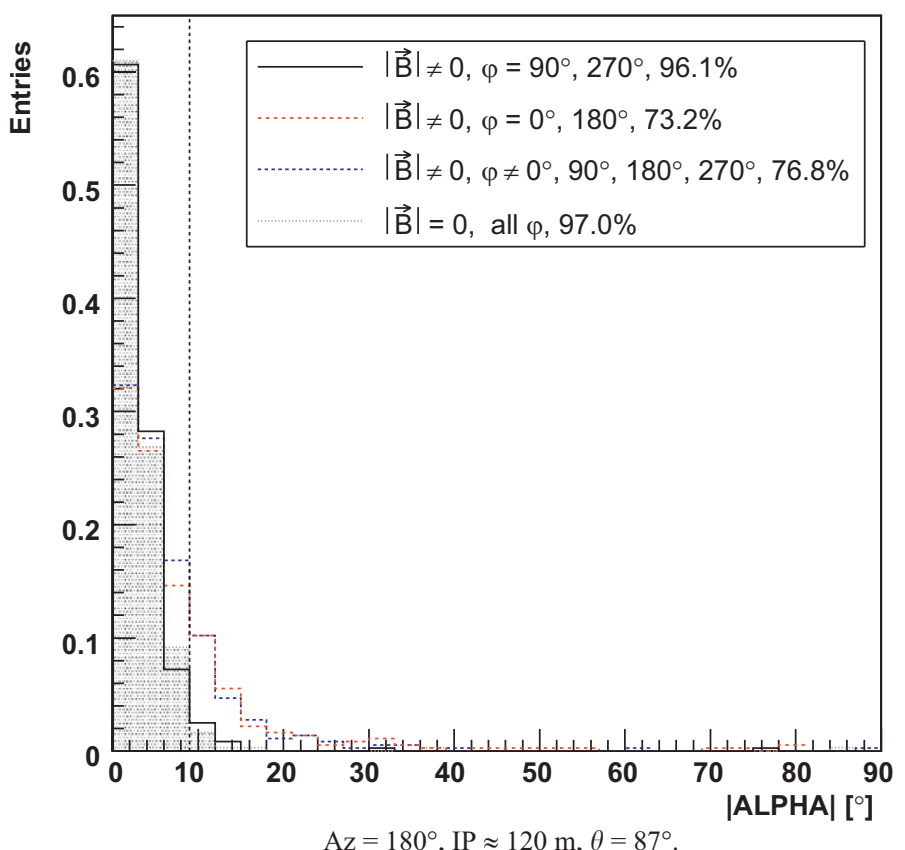

Fig. 9. As Fig. 8, but for $450 \mathrm{GeV} \gamma$-rays, $\mathrm{ZA}=40^{\circ}, \mathrm{Az}=0^{\circ}$ and $180^{\circ}$.

was set to $120 \mathrm{~m}$. The angle $\theta$ between the direction of the EAS and the GF is given on top of the abscissa. The $\gamma$ efficiency is defined as the ratio of the number of $\gamma$-ray showers surviving the trigger and the image cleaning to the number of generated $\gamma$-rays. From Fig. 14(d) it can be seen that the refficiency varies by up to $50 \%$. For showers close to the trigger threshold the Cherenkov light distribution on the ground can be thinned out such that most of the events do not survive the trigger level. This occurs only for showers close to the energy threshold of the telescope, which is ZA dependent.

For some telescope pointing directions the total reconstructed integrated light of shower images can be reduced by up to $\sim 20 \%$. This is not only the case for low energies but also for $\mathrm{TeV} \gamma$-rays.
Consequently, if GF effects are not taken into account the energy of $\gamma$-ray candidates from observational data will be systematically underestimated whereas the $\gamma$ efficiency will be overestimated. Both effects degrade the determination of the flux from a $\gamma$-ray source if they are not properly taken into account in the MC simulation.

\section{Conclusions}

The results from the MC studies show that the GF can significantly affect both the shape and the orientation of shower images recorded with an IACT like MAGIC. Therefore, the 
a

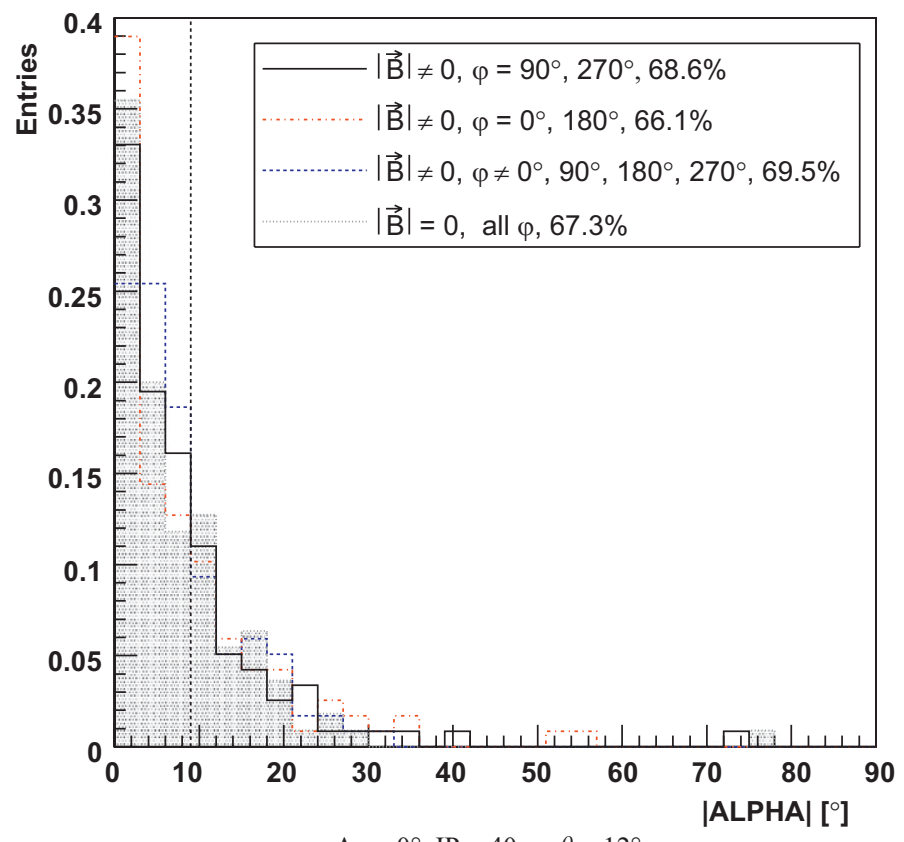

C

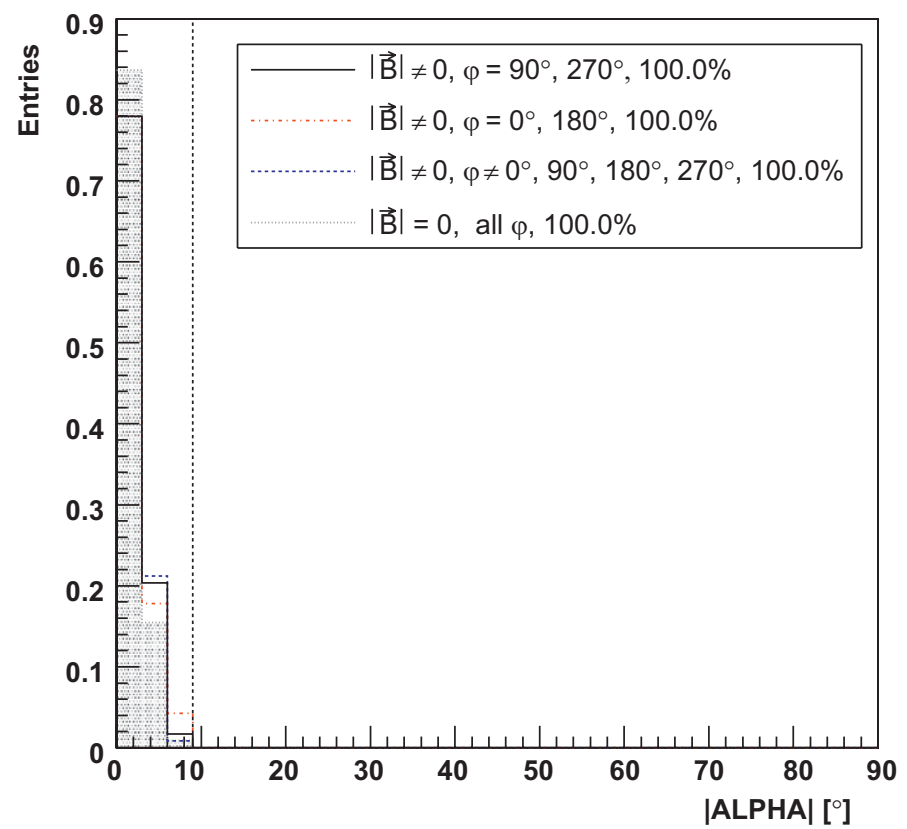

b

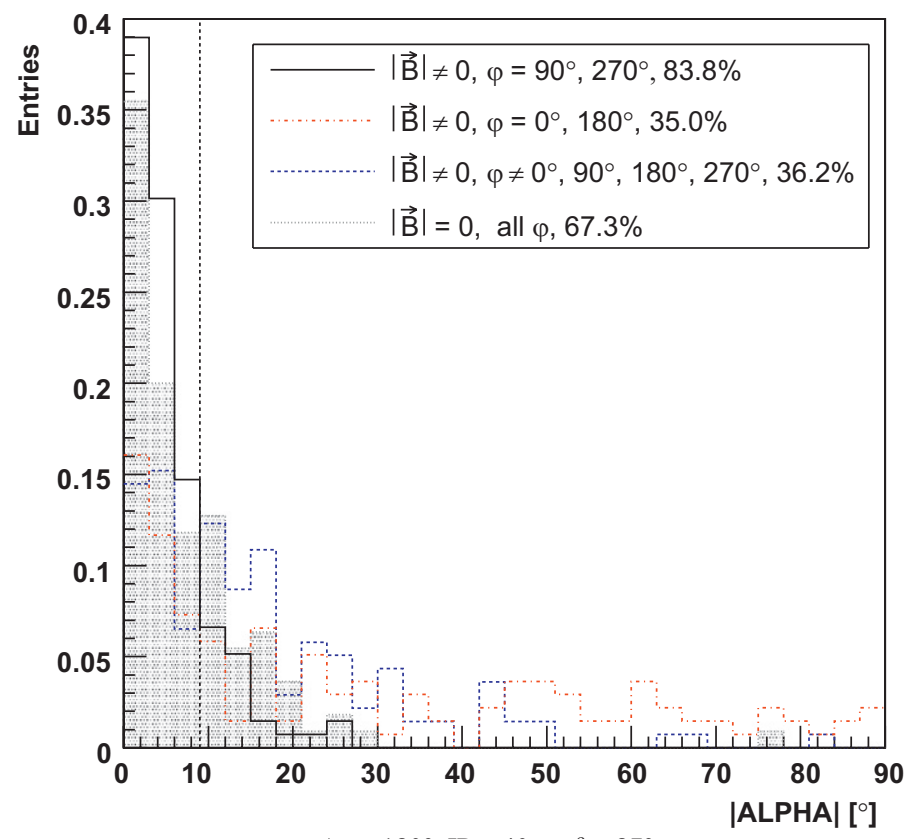

$\mathrm{Az}=180^{\circ}, \mathrm{IP} \approx 40 \mathrm{~m}, \theta=87^{\circ}$.

d

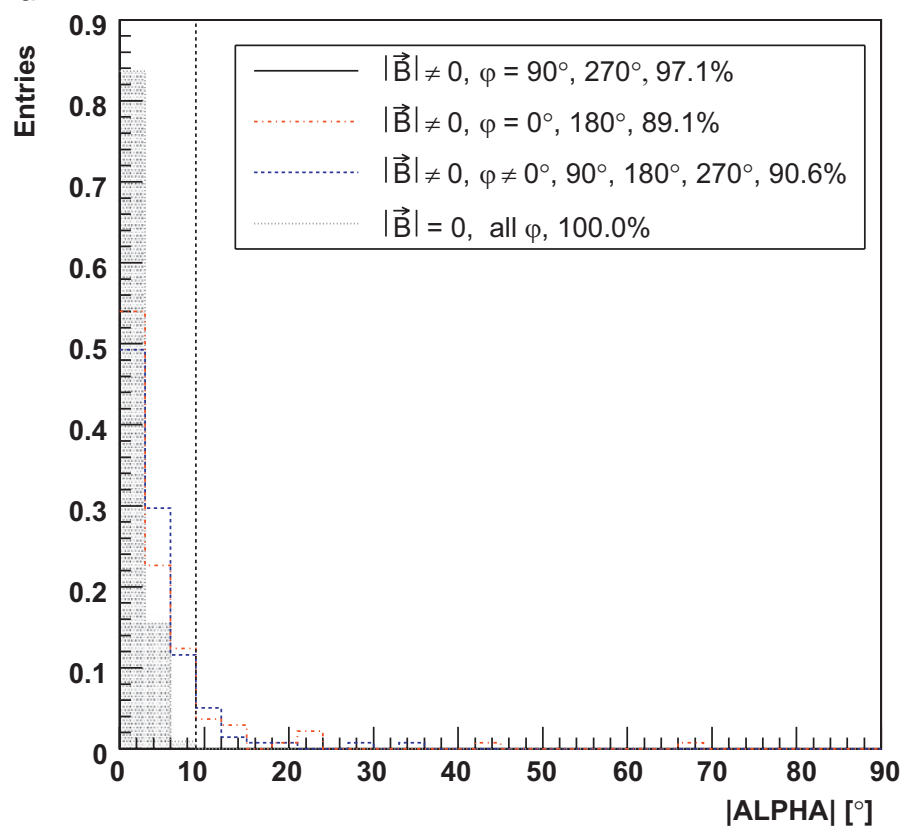

$\mathrm{Az}=180^{\circ}, \mathrm{IP} \approx 120 \mathrm{~m}, \theta=87^{\circ}$.

Fig. 10. As Fig. 8, but for $1 \mathrm{TeV} \gamma$-rays, $\mathrm{ZA}=40^{\circ}, \mathrm{Az}=0^{\circ}$ and $180^{\circ}$.

orientation discrimination of $\gamma$-rays against unwanted (hadronic) background can be significantly degraded. It was demonstrated that the de-rotation of the shower images does not help to recover the pointing entirely. At most $10 \%$ of the events can be recovered by de-rotation requiring the knowledge of the impact parameter and energy of the $\gamma$-rays.

The influence of the GF also degrades the DISP-estimated arrival direction of MC-generated $\gamma$-rays. Due to the influence of the GF on the development of EAS the DISP distribution can be significantly elongated perpendicular to the projection of the GF in the camera. The quality of a sky map is degraded in a way that a point-like source appears extended unless it is compared to a proper MC simulation taking into account the trajectory of the source in the sky. However, the peak of the DISP distribution is always centred at the source position.

It was also shown that the influence of the GF on EAS can significantly affect the energy reconstruction and the trigger efficiency for $\gamma$-rays. If this effect is not taken into account, the energy of $\gamma$-ray candidates from observational data will be systematically underestimated (up to $\sim 20 \%$ effect). For low energies close to the analysis threshold $(<100 \mathrm{GeV})$ the $\gamma$ efficiency also depends on the position of the telescope in the 
a

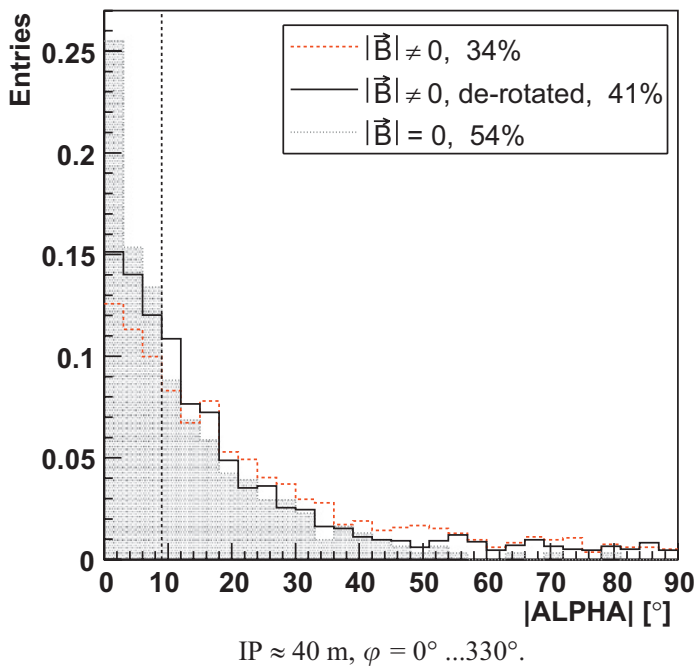

C

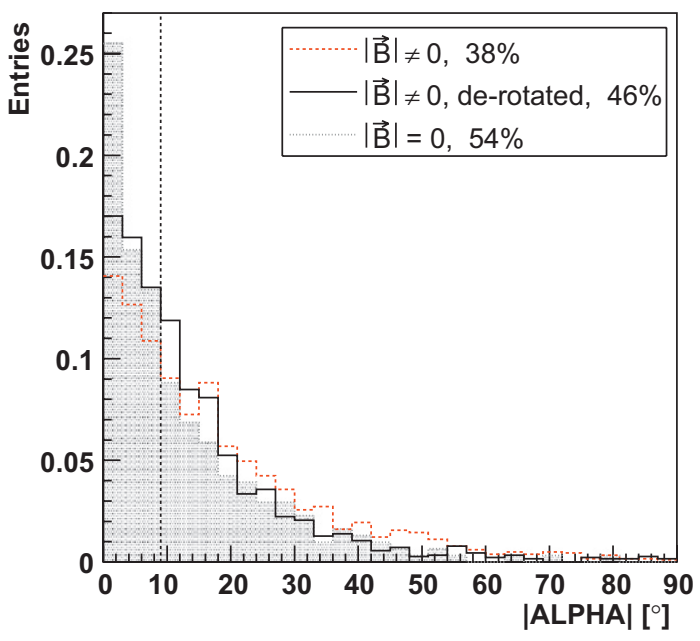

$\mathrm{IP} \approx 40 \mathrm{~m}, \varphi=0^{\circ}$ and $180^{\circ}$.

e

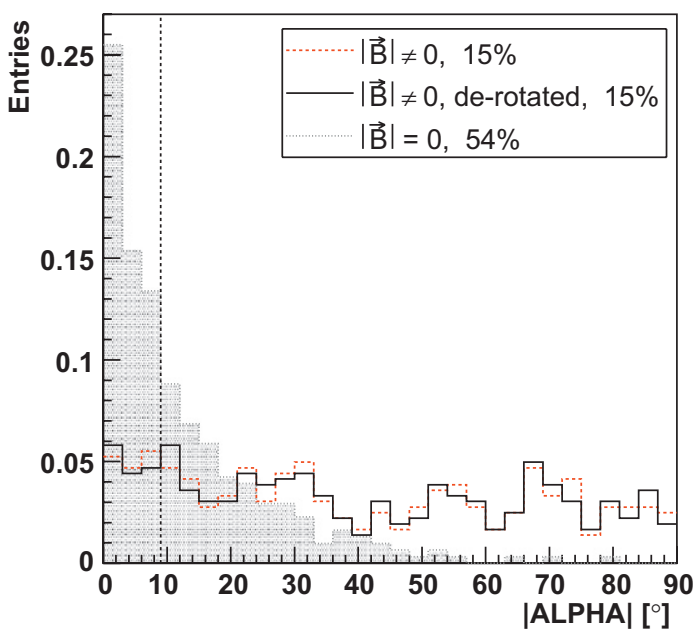

$\mathrm{IP} \approx 40 \mathrm{~m}, \varphi=0^{\circ}$ and $180^{\circ}$. b

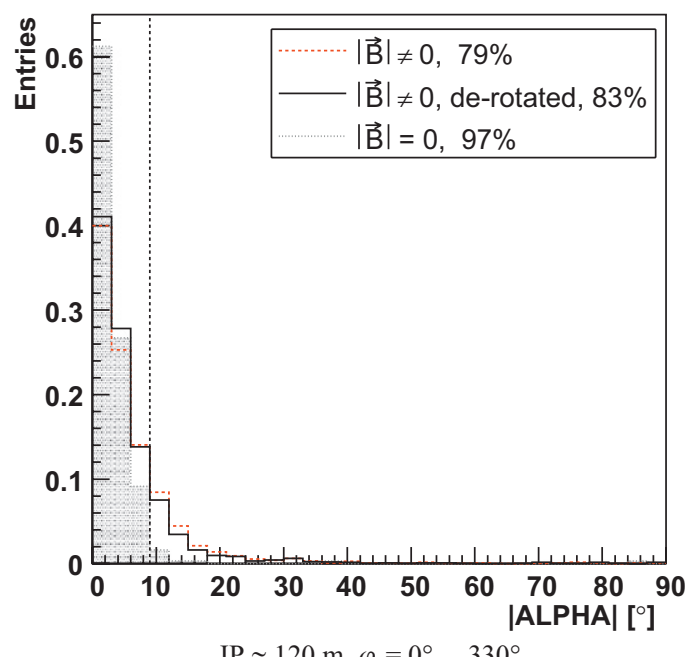

d

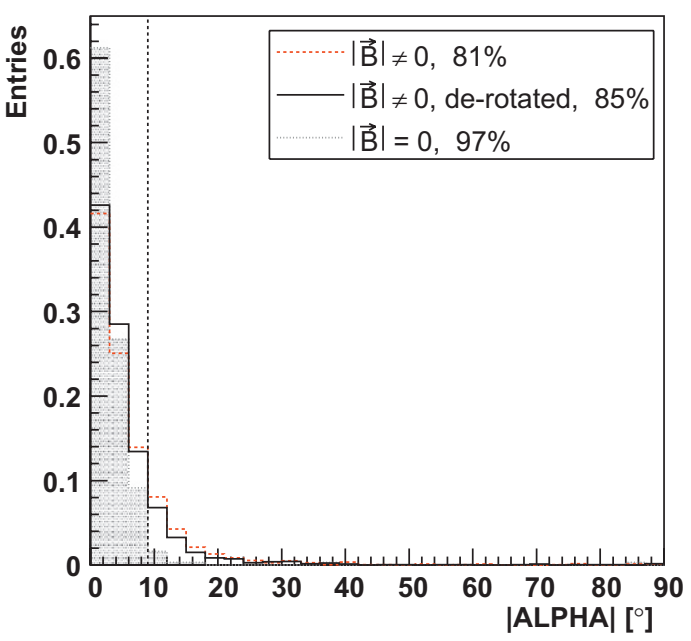

$\mathrm{IP} \approx 120 \mathrm{~m}, \varphi=0^{\circ}$ and $180^{\circ}$.

f

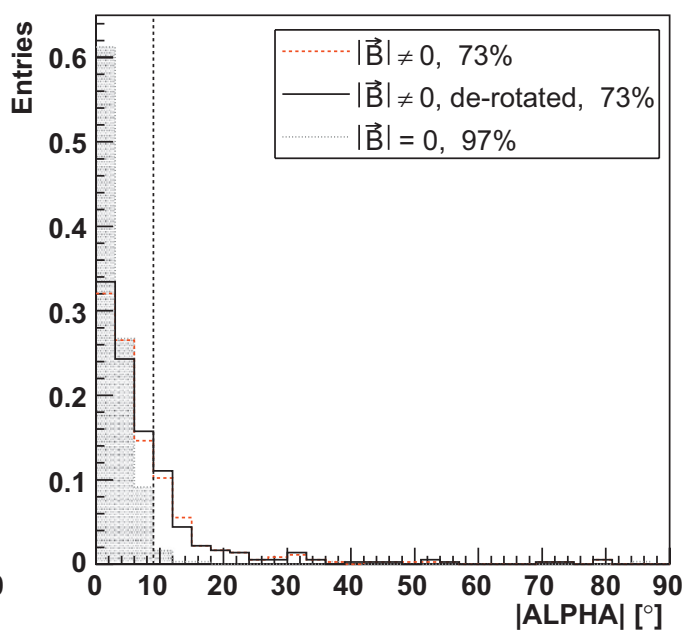

$\mathrm{IP} \approx 120 \mathrm{~m}, \varphi=0^{\circ}$ and $180^{\circ}$

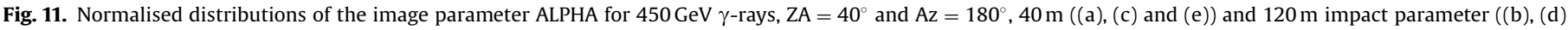

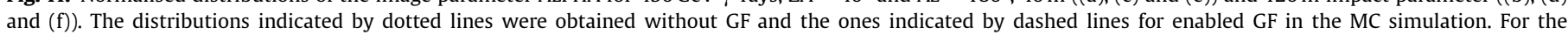
distributions indicated by solid lines the shower images were de-rotated (see text for more details). 
a

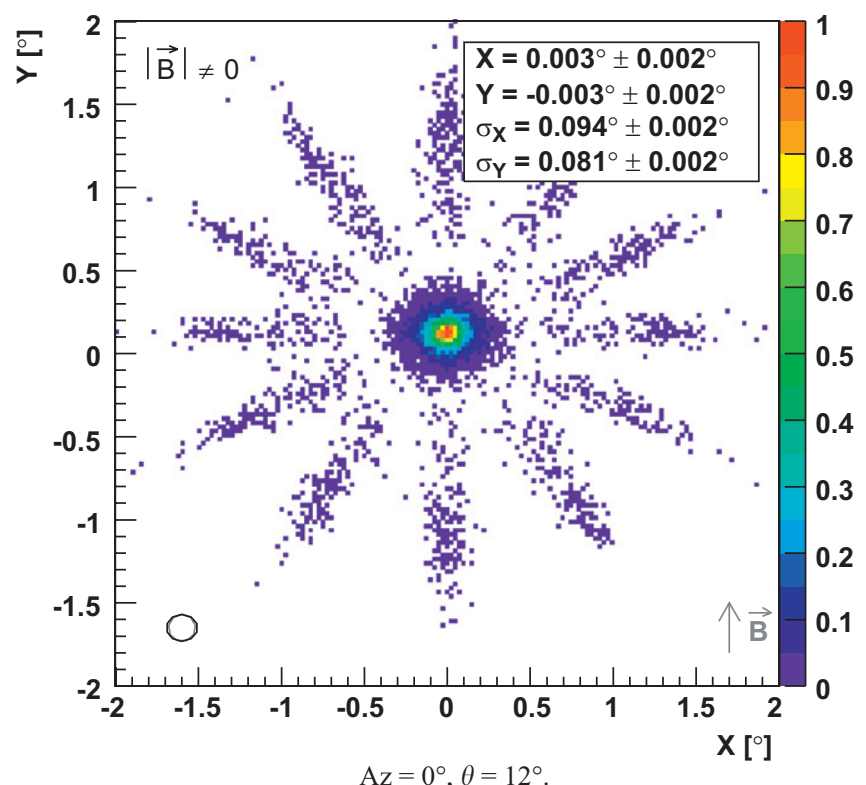

b

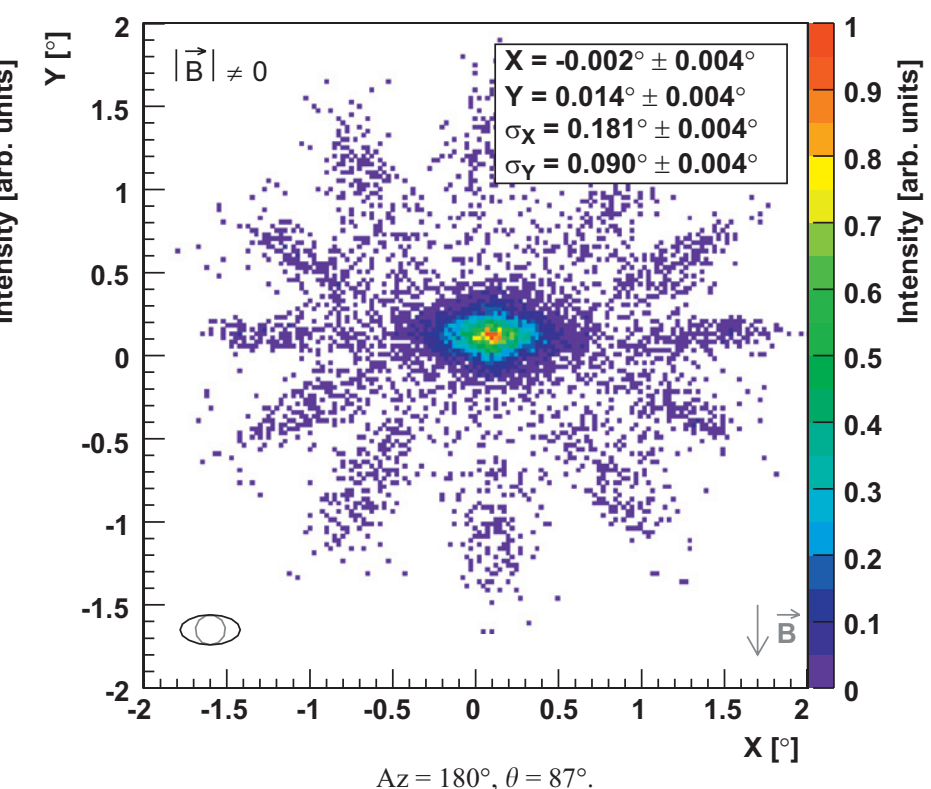

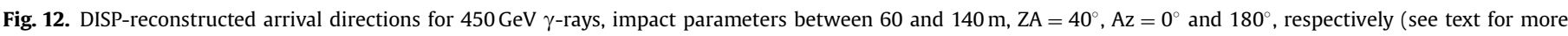
details).

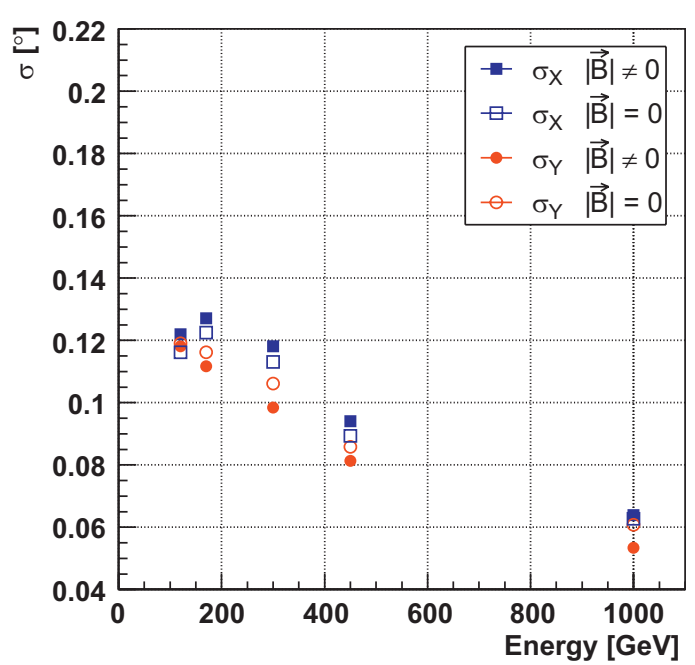

$\mathrm{Az}=0^{\circ}, \mathrm{ZA}=40^{\circ}, \theta=12^{\circ}$.

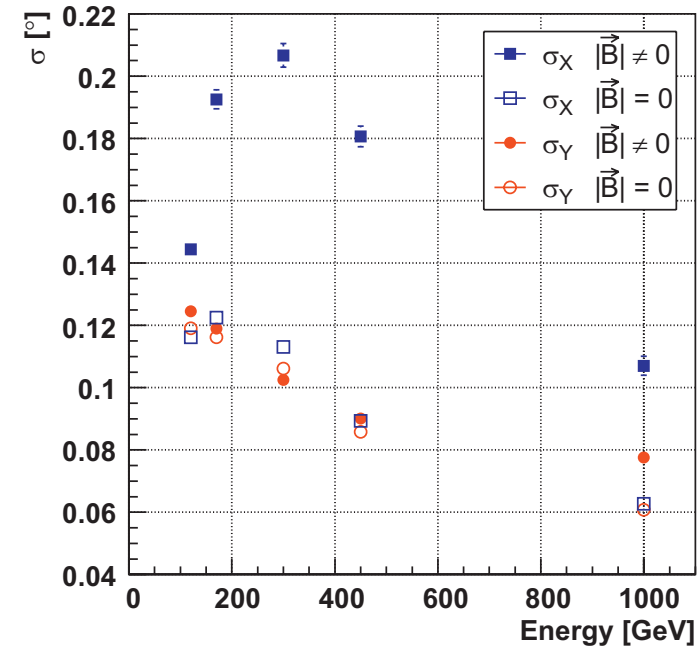

$\mathrm{Az}=180^{\circ}, \mathrm{ZA}=40^{\circ}, \theta=87^{\circ}$.

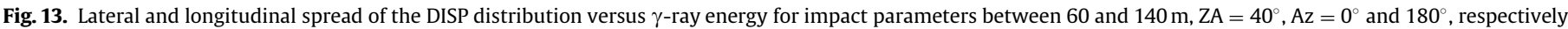
(see text for more details).

Cherenkov light pool [1]. At higher energies ( $300 \mathrm{GeV}-1 \mathrm{TeV})$, the $\gamma$ efficiency is affected only at large ZA, where the telescope threshold energy is significantly increased ( $\$ 50 \%$ effect).

It was demonstrated that the extent of the GF effects not only depends on the orientation of EAS with respect to the direction of the GF but also on the position of the telescope with respect to the EAS core location on ground. Shower images are not only rotated away from the projected direction of the GF in the telescope camera plane but can also be rotated towards it, contrary to what was reported in Ref. [8].

Altogether, GF effects on EAS affect the $\gamma$-ray sensitivity of an IACT and the determination of the flux from a VHE $\gamma$-ray source. Distinct MC data covering the same $\mathrm{ZA}$ and $\mathrm{Az}$ angle range as the observational data being analysed are required to account for GF effects.
It is remarkable that the GF effects not only occur at very low energies but also at high energies around $1 \mathrm{TeV}$. The GF effects are rather pronounced at $\gamma$-ray energies around $450 \mathrm{GeV}$. The reason for GF effects to occur at high energies is presumably linked to a characteristic feature in the development of a $\gamma$-rayinduced EAS. The process of multiplication in EAS continues until the average energy of the shower particles is insufficient to further produce secondary particles in subsequent collisions. At this stage of the shower development, the shower maximum is reached (largest number of secondary particles) and the average energy of the secondaries is close to the so-called critical energy of $\sim 100 \mathrm{MeV}[26]$ below which secondary electrons and positrons lose their energy predominantly through ionisation of air molecules [27]. At the shower maximum, the average energy of the secondary particles is independent of the primary $\gamma$-ray 
a

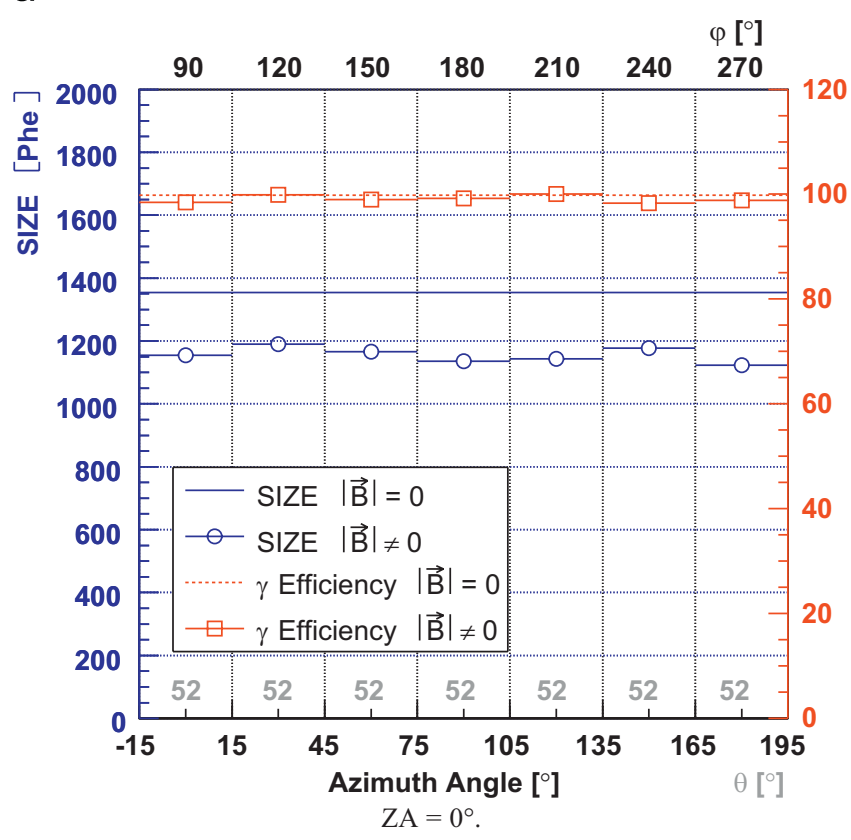

C

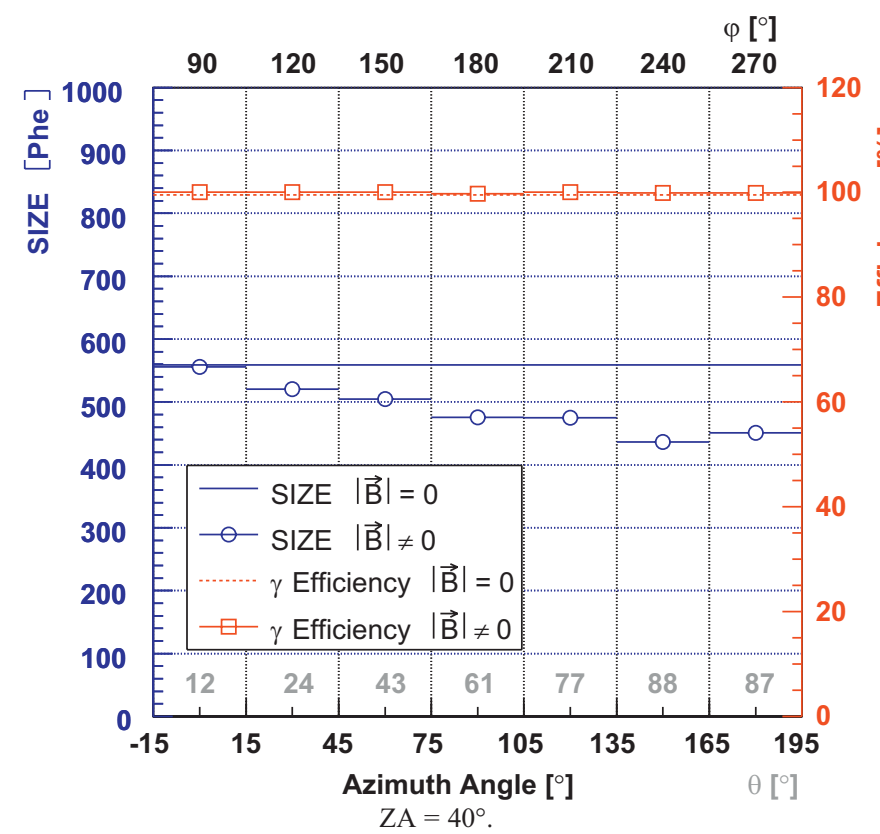

b

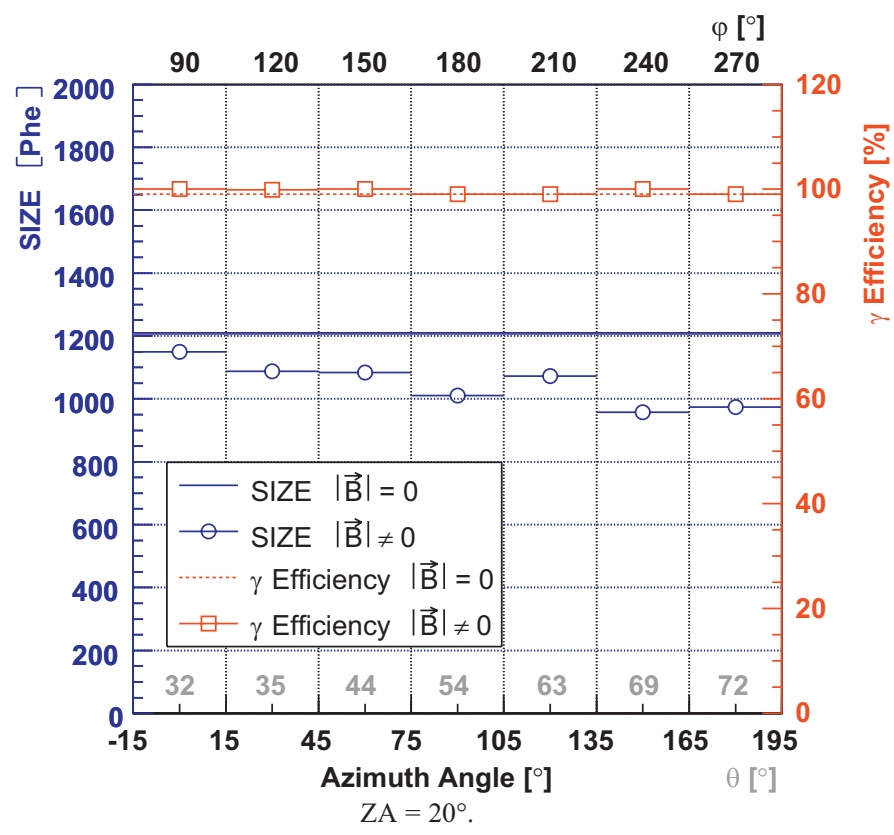

d

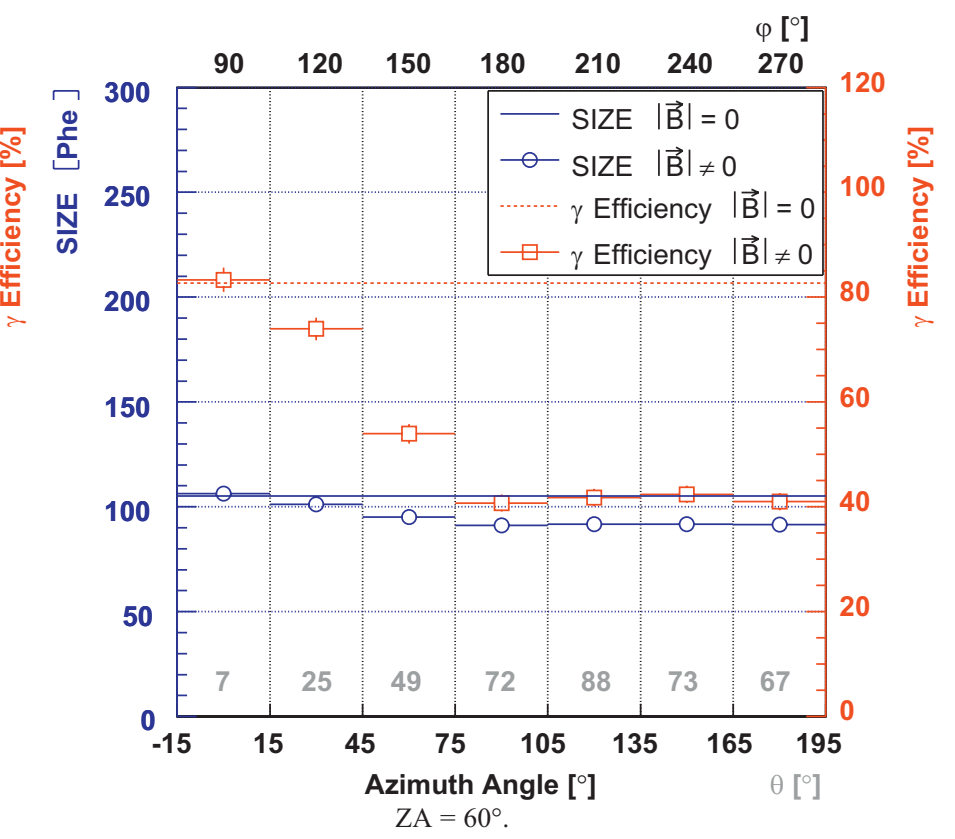

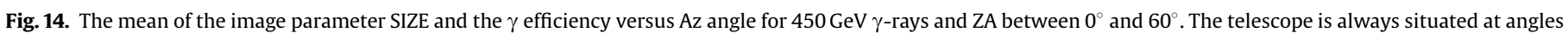
$\varphi=\mathrm{Az}+90^{\circ}$ and $120 \mathrm{~m}$ impact parameter.

energy and the GF has on average the same influence on the secondary particles.

Apart from that, the average atmospheric depth at which the shower maximum occurs increases logarithmically with increasing energy of the primary $\gamma$-ray [27], and therefore the track along which secondary electrons and positrons suffer from Lorentz deflection increases, too.

Another point worthy of mentioning is the fact that the threshold energy for a charged particle to emit Cherenkov light decreases with increasing atmospheric depth. Hence, in highenergy EAS, even charged secondaries of lower energy suffering strong Lorentz deflection may additionally contribute to the Cherenkov light pool on ground.
GF effects on the hadron-induced background were not studied. It is impossible to show the rotation effect using shower images from hadron candidates of observational data, because they do not point to any source. Also, possible GF effects on the hadroninduced background presumably do not degrade the background discrimination. Close to the energy threshold the trigger efficiency for the hadronic background should be reduced, too.

\section{Outlook}

Although this study focuses on GF effects relevant for a single IACT like MAGIC the influence of the GF on the development of 
EAS is expected to degrade also the performance of stereoscopic IACT arrays. In a stereoscopic telescope system multiple telescopes view the same EAS. The individual images are then combined to form a common event. Therefore, stereoscopic systems allow for a three-dimensional reconstruction of the shower axis resulting in an improved sensitivity. The shower direction on the sky is estimated from the intersection point of the major image axes in a composite field of view, on an event by event basis [22]. Due to the rotation of the individual shower images the intersection point will be modified in a way that the source direction in the sky is wrongly reconstructed. Stereoscopic systems do not trigger homogeneously but preferably on EAS with impact positions between the telescopes. As the impact distance on ground between the EAS and the individual telescopes of an array is different in the majority of cases, the GF will deteriorate the orientation of the individual shower images differently. As a result the performance of stereoscopic IACT arrays is degraded by GF effects. A detailed MC study on the influence of GF effects on the performance of stereoscopic IACT systems is in preparation.

The intensity of the GF on the Earth' surface ranges from about $20 \mu \mathrm{T}$ to about $70 \mu \mathrm{T}$ [17]. It is therefore important to take into account the GF effects for the site selection of future projects utilising the imaging air Cherenkov technique. To minimise the influence of the GF on the detector performance it is mandatory to select a site with a low absolute value of the GF. Hence, the bestsuited location would be close to the so-called South Atlantic Anomaly, where the GF strength is minimal, amounting to about one half of the value for the MAGIC telescope site.

It is difficult to study GF effects in observational data. The elevation effect on shower images complicates such studies. Directions with strong magnetic field correspond to large ZA and the sensitivity of an IACT changes as a function of the ZA as a result of changing shower image characteristics due to increasing air mass with increasing ZA [22]. There are several requirements a $\gamma$-ray source should fulfil to be an appropriate candidate for GF studies in observational data: it should be strong, preferably point-like, stable and it should follow a trajectory corresponding to a large GF component normal to the telescope pointing direction (Fig. 1(a)).

Preliminary results from studies on GF effects in observational data taken with MAGIC were already shown in Refs. [1,28]. It was demonstrated that the pointing resolution of MAGIC allows to study GF effects in observational data even for a very low component of the GF normal to the shower direction. However, an extensive study on GF effects in observational data is in progress.

\section{References}

[1] S.C. Commichau, Observation of very high energy gamma-rays from the galactic center with the MAGIC Telescope considering geomagnetic field effects on the imaging technique, Ph.D. Thesis (No. 17118), ETH Zürich, 2007.

[2] A. Wenger, Diploma Thesis, The effect of the earth's magnetic field on extensive air showers, ETH Zürich, 2004

[3] R. de los Reyes-Lopez R, Ph.D. Thesis, UC Madrid, 2008.

[4] G. Cocconi, Phys. Rev. 93 (1954) 646.

[5] N.A. Porter, Nuovo Cimento Lett. 8 (1973) 481.

[6] C.C.G. Bowden, et al., J. Phys. G: Nucl. Part. Phys. 18 (1992) L55.

[7] M.J. Lang, et al., J. Phys. G: Nucl. Part. Phys. 20 (1994) 1841.

[8] P.M. Chadwick, et al., J. Phys. G: Nucl. Part. Phys. 25 (1999) 1223.

[9] P.M. Chadwick, Geomagnetic effects on the performance of atmospheric Cherenkov telescopes, in: Proceedings of the 26th International Cosmic Ray Conference, vol. 5, 1999, p. 231.

[10] P.M. Chadwick, et al., J. Phys. G: Nucl. Part. Phys. 26 (2000) L5.

[11] K.M. Aye, et al., Correcting high resolution imaging for the effects of the geomagnetic field, in: Proceedings of the 27th International Cosmic Ray Conference, vol. 7, 2001, p. 2842.

[12] E. Lorenz, New Astron. Rev. 48 (2004) 339.

[13] J.A. Barrio, et al., The MAGIC telescope design report, MPI Institute Report MPI-PhE/98-5, Munich, 1998.

[14] F. Goebel, MAGIC Collaboration, et al., in: Status of the second phase of the MAGIC telescope, Proceedings of the 30th International Cosmic Ray Conference, Merida, 2007.

[15] J. Albert, MAGIC Collaboration, et al., Astrophys. J. 674 (2008) 1037.

[16] D. Heck, et al., Report FZKA 6019 (1998), Forschungszentrum Karlsruhe 〈 http://www-ik.fzk.de/corsika/〉.

[17] National Geographic Data Center (NGDC) 〈http://www.ngdc.noaa.gov/geomag/〉.

[18] P. Majumdar, MAGIC Collaboration, et al., Monte Carlo simulation for the MAGIC telescope, in: Proceedings of the 29th International Cosmic Ray Conference, vol. 5, 2005, p. 203.

[19] A.M. Hillas, Cherenkov light images of EAS produced by primary gamma rays and by nuclei, in: Proceedings of the 19th International Cosmic Ray Conference, vol. 3, 1985, p. 445.

[20] T. Bretz, R.M. Wagner, MAGIC Collaboration, in: The MAGIC Analysis and Reconstruction Software, Proceedings of the 28th International Cosmic Ray Conference, vol. 5, Elsevier, NJ, 2003, p. 2947.

[21] R. Mirzoyan, E. Lorenz, Measurement of the night sky light background at La Palma, MPI Institute Report MPI-PhE/94-35, Munich, 1994

[22] D.J. Fegan, J. Phys. G: Nucl. Part. Phys. 23 (1997) 1013.

[23] V.P. Fomin, et al., Astropart. Phys. 2 (1994) 137.

[24] R.W. Lessard, et al., Astropart. Phys. 15 (2001) 1

[25] E. Domingo-Santamaría, MAGIC Collaboration, et al., in: The DISP analysis method for point-like or extended $\gamma$ source searches/studies with the MAGIC Telescope, Proceedings of the 29th International Cosmic Ray Conference, vol. 5, 2005, p. 363.

[26] H. Bethe, W. Heitler, Proc. R. Soc. 146 (1934) 83.

[27] K. Greisen, Progr. Cosmic Ray Phys. 3 (1956) 27.

[28] S.C. Commichau, MAGIC Collaboration, et al., in: Geomagnetic field effects on the imaging air shower Cherenkov technique, Proceedings of the 30th International Cosmic Ray Conference, Merida, 2007. 